\title{
A Robust MRAC Using Variable Structure Design for Multivariable Plants*
}

\author{
CHIANG-JU CHIEN, $\dagger$ KING-CHUAN SUN, $\ddagger$ A-CHEN WU $\ddagger$ and LI-CHEN FU
}

\begin{abstract}
The variable structure design concept has been successfully incorporated to establish a model reference adaptive controller for multivariable plants with arbitrary generalized relative degree and with the aim of achieving global stability, good robustness and well-behaved tracking performances.
\end{abstract}

\begin{abstract}
Key Words-Robustness; model reference adaptive control; multivariable plants; arbitrary generalized relative degree; variable structure design.
\end{abstract}

\begin{abstract}
It is well known that undesirable transient responses and tracking performances have been frequently observed in traditional model reference adaptive control (MRAC) problems, especially for multivariable plants with unmodeled dynamics and output disturbances. In this paper, a new robust model reference adaptive control using variable structure design (VSD) is proposed to investigate the solution. Based on a definition of generalized relative degree (GRD) for multivariable plants, the general case for plants with arbitrary GRD is completely solved. It is shown that, even when the uncertainties are presnet, global stability and robustness of the closed-loop control system are achieved. Furthermore, without any persistence of excitation, the tracking errors will, at least asymptotically, converge to zero for GRD-one plants and to a small residual set for plants with any higher GRD. With a suitable choice of initial control parameters, the tracking errors can even be driven to zero in finite time for GRD-one plants and to a small residual set exponentially for plants with any higher GRD.
\end{abstract}

Copyright (C) 1996 elsevier Science Ltd.

\section{INTRODUCTION}

With advances in designing adaptive controllers for single-input single-output (SISO) dynamical systems, a multi-input multi-output (MIMO) model reference adaptive control system has been proposed by Elliott and Wolovich (1982). Since an important problem for parameterization of an MIMO plant in the context of MRAC is the determination of its interactor matrix (Wolovich and Falb, 1976), some research has been conducted on how to use less a priori

\footnotetext{
* Received 19 October 1992; revised 11 September 1993; revised 18 October 1994; revised 25 September 1995; received in final form 6 November 1995. This paper was not presented at any IFAC meeting. This paper was recommended for publication in revised form by Editor C. C. Hang. Corresponding author Professor Chiang-Ju Chien. Tel. +88626632102/6632106; Fax +8862 6631119; E-mail cjc@ee.hcht.edu.tw.

† Department of Electronic Engineering, Hua Fan College of Humanities and Technology, Shihtin, Taipei Hsien, Taiwan, Republic of China.

$\ddagger$ Department of Electrical Engineering, National Taiwan University, Taipei, Taiwan, Republic of China.
}

knowledge of the interactor matrix to design an adaptive controller (Das, 1986; Dion et al., 1988) and or how to decide the interactor matrix from information on the relative degree of each entry of the MIMO transfer matrix (Singh and Narendra, 1984; Asano et al., 1990). However, the robustness problem and/or tracking performance are not addressed in the above work. Hence another line of research, such as that by Tao and Ioannou (1988), is focused on the development of the MRAC scheme for MIMO systems with unmodeled dynamics and output disturbances. Solving the problem of unpredictable transient response and tracking performance has recently become one of the challenging aspects of MRAC. A considerable amount of effort has been made to improve these schemes to obtain better control responses. It should be noted that the variable structure design (VSD) or switching mechanism with high-gain feedback is now frequently used to design controllers or update laws for SISO plants (Hsu and Costa, 1989; Hsu, 1990; Fu, 1991; Narendra and Bơsković, 1992; Miller and Davison, 1991) and a class of MIMO plants (Tao and Ioannou, 1989; Chien and Fu, 1992) in order to overcome these difficulties. For SISO linear time-invariant systems, the so-called VS-MRAC scheme, first proposed by Hsu and Costa (1989), incorporates switching on the adjustable parameters $\theta$ to achieve tracking performance. The robustness problem of the VS-MRAC scheme was discussed by Costa and Hsu (1992). The later work by Fu (1991) applied switching not only to parameter adaptation but also to plant control input with the consideration of unmodeled dynamics and output disturbances. However, only plants of relative degree one are 
considered in the above papers. Owing to the assumption that only input-output measurement is available, the extension of the abovementioned adaptive variable structure design to that for systems with arbitrary relative degree is difficult. A special case for plants of relative degree two with uncertainties was discussed by Fu (1992), who obtained good tracking performance. On the other hand, the VS-MRAC scheme was extended to the general case of plants of arbitrary relative degree by Hsu (1990); however, the robustness issue was not addressed and the crucial assumption that 'equivalent control' is measurable was overly restrictive. Furthermore, a combined direct, indirect and variable structure design approach can be found in the work by Narendra and Borsković (1992). For MIMO linear time-invariant plants, relatively fewer results are found in this field. The first work to study the robustness of MIMO MRAC schemes by using variable structure design was that of Tao and Ioannou (1989). Later, Chien and Fu (1992) introduced the variable structure concept into the design of controller for MIMO plants in order to guarantee the robustness and performance of the closed-loop system. However, both results are not for the general case, and are limited to a class of multivariable plants.

In this paper, some prior information for multivariable plants, such as the interactor matrix, is used to develop a new adaptive variable structure scheme for solving problems with global stability, robustness, tracking and transient performances existing in traditional MRAC systems. By a definition of generalized relative degree (GRD) for multivariable plants, the adaptive variable structure controllers here are then designed for the arbitrarily GRD case (the so-called general case; Hsu, 1990). Compared with previous work, which used adaptive variable structure design or the traditional robust adaptive approaches for the linear MRAC problem, this paper has the following special features.

(i) This is the first work that applies the adaptive variable structure design for multivariable unknown plants in the general case under the robustness consideration.

(ii) The control strategy using the concept of 'average control' rather that that of 'equivalent control' is thoroughly analyzed.

(iii) A systematic design approach is proposed for multivariable plants with arbitrary GRD, and a new adaptation mechanism is developed so that prior upper bounds on some appropriately defined but unavailable system parameters are not needed. It is shown that, without any persistent excitation, global stability and robustness with asymptotic tracking performance can be guaranteed. The output tracking errors can be driven to zero for GRD-one systems and to a small residual set (whose size depends on the level of magnitude of some design parameter) for systems with any higher GRD. Both results are achieved even when unmodeled dynamics and output disturbances are present.

(iv) If the aforementioned bounds on the system parameters are available by some means before controller design then, with a suitable choice of initial control parameters, the output tracking errors can even be driven to zero in finite time for GRD-one systems and to a small residual set exponentially for systems with any higher GRD. It should be noted that these bounds are usually assumed to be known before the construction of the variable structure controller (Hsu, 1990) or the robust adaptation law (switching $\sigma$ modification; Tao and Ioannou, 1988).

In order to make a comparison between the proposed adaptive variable structure scheme and the traditional approaches, we choose the robust adaptive MRAC scheme with switching $\sigma$ modification (Tao and Ioannou, 1988) for computer simulations, since this is the only work that successfully solves the general case of the robust MRAC problem for MIMO plants to date. The differences in tracking performance between these two schemes will be easily observed from the simulation results, and some remarks about the advantages of our design will also be made.

The theoretical framework in this paper is developed on the basis of Filippov's (1964) solution concept for differential equation with discontinuous right-hand side. In the subsequent discussion, the followng notation will be used.

(i) $G(s)[u](t)$ denotes the filtered version of $u(t)$ with a proper or strictly proper transfer function (matrix) $G(s)$.

(ii) $|\cdot|$ denotes the absolute value of a scalar or the Euclidean norm of a vector or matrix.

(iii) $\left\|(\cdot)_{t}\right\|_{\infty}=\sup _{\tau \leq l}|(\cdot)(\tau)|$ denotes the truncated $L_{\infty}$ norm of the argument function or vector.

(iv) $\|G(s)\|_{\infty}$ denotes the $H_{x}$ norm of the transfer function $G(s)$. 
The paper is organized as follows. In Section 2 , we give a detailed problem formulation including plant parameterization, control objective, plant assumptions and definition of GRD, and derive a suitable error equation between the plant and reference model. Based on the concept of GRD, a robust adaptive variable structure controller is proposed in Section 3 for multivariable systems with GRD equal to one. An extension of this controller design concept to systems with GRD greater than one is presented in Section 4. Section 5 gives simulation results of an aircraft model to demonstrate the effectiveness of the adaptive variable structure controller, which is compared with a traditional robust adaptive approach. Finally, conclusions are drawn in Section 6.

\section{PROBLEM FORMULATION}

\subsection{Plant description and MRI matrix}

The interactor matrix (Wolovich and Falb, 1976; Elliott and Wolovich, 1982, 1984; Das, 1986; Dion et al., 1988; Tao and Ioannou, 1988) or the Hermite normal form (Singh and Narendra, 1984) plays an important role in parameterizing MIMO plants in the context of MRAC for either continuous-time or discretetime systems. In this paper, however, the focus is on how to design an adaptive variable structure control scheme such that global stability is guaranteed in the presence of unmodeled dynamics and output disturbances and such that the tracking performance is improved compared with traditional MRAC systems. To suit our purpose, we choose the concept of modified right interactor (MRI) matrix (Tao and Ioannou, 1988) for plant parameterization in the following discussion.

Consider an MIMO linear time-invariant plant with $n$ inputs and $n$ outputs described by the equation

$$
\begin{aligned}
\hat{y}_{\mathrm{p}}(t) & =P_{\mathrm{o}}(s)\left[I+\mu \Delta P_{\mathrm{u}}(s)\right]\left[u_{\mathrm{p}}\right](t)+d_{\mathrm{o}}(t) \\
& \equiv y_{\mathrm{p}}(t)+d_{\mathrm{o}}(t) .
\end{aligned}
$$

where $P_{\mathrm{o}}(s)$ is the strictly proper rational transfer matrix of the nominal plant, $\mu \Delta P_{\mathrm{u}}(s)$ is the multiplicative unmodeled dynamics with some $\mu \in \mathbb{R}^{+}$, and $d_{\mathrm{o}} \in \mathbb{R}^{n}$ is the output disturbance. It has been shown (Tao and Ioannou, 1988) that if the nominal plant $P_{\mathrm{o}}(s)$ (i.e. when $\mu=0, d_{\mathrm{o}}=0$ ) is strictly proper rational with full rank then there exists an upper-triangular polynomial matrix $\xi_{\mathrm{r}}(s)$ defined as the MRI matrix of $P_{\mathrm{o}}(s)$ such that

$$
\lim _{s \rightarrow \infty} P_{\mathrm{o}}(s) \xi_{\mathrm{r}}(s)=K_{\mathrm{r}},
$$

where $K_{\mathrm{r}}$ is nonsingular. Let $S_{\mathrm{r}}$ be a matrix such that $K_{\mathrm{r}} S_{\mathrm{r}} \triangleq \Gamma_{\mathrm{r}}$ is positive-definite. Then the following lemma will employ the notion of the MRI matrix to give a parameterization of the plant $P_{\mathrm{o}}(s)$ that is useful in designing MRAC schemes.

Lemma 2.1. The MIMO linear time-invariant plant

$$
y_{\mathrm{p}}(t)=P_{\mathrm{o}}(s)\left[u_{\mathrm{p}}\right](t),
$$

with $P_{\mathrm{o}}(s)$ strictly proper rational with full rank, may be represented as

$$
\begin{aligned}
u_{\mathrm{p}}(t) & =\frac{1}{f_{\mathrm{r}}(s)} I \xi_{\mathrm{r}}(s) S_{\mathrm{r}}[v](t), \\
y_{\mathrm{p}}(t) & =P_{\mathrm{o}}(s) \frac{1}{f_{\mathrm{r}}(s)} I \xi_{\mathrm{r}}(s) S_{\mathrm{r}}[v](t) \\
& \triangleq P_{\mathrm{r}}(s)[v](t),
\end{aligned}
$$

where $\xi_{\mathrm{r}}(s)$ is the MRI matrix of $P_{\mathrm{o}}(s)$ with high-frequency gain matrix $K_{\mathrm{r}}$, and $f_{\mathrm{r}}(s)$ is an arbitrary Hurwitz polynomial whose degree $\rho$ is equal to the maximum degree of the elements of $\xi_{\mathrm{r}}(s)$. Furthermore, the MRI matrix of $P_{\mathrm{r}}(s)$ is the diagonal matrix $f_{\mathrm{r}}(s) I$, and the highfrequency gain matrix of $P_{\mathrm{r}}(s)$ is equal to $K_{\mathrm{r}} S_{\mathrm{r}} \triangleq \Gamma_{\mathrm{r}}$.

\subsection{Control objective, plant assumptions and generalized relative degree}

By the parameterization of the nominal plant $P_{\mathrm{o}}(s)$ described in Lemma 2.1, we can now rewrite (1) as

$$
\begin{aligned}
& \hat{y}_{\mathrm{p}}(t)=G_{\mathrm{r}}(s)[v](t)+d_{\mathrm{o}}(t), \\
& u_{\mathrm{p}}(t)=\frac{1}{f_{\mathrm{r}}(s)} l \xi_{\mathrm{r}}(s) S_{\mathrm{r}}[v](t),
\end{aligned}
$$

where

$$
G_{\mathrm{r}}(s)=P_{\mathrm{r}}(s)\left[I+\mu \Delta P_{\mathrm{ur}}(s)\right],
$$

with

$$
\begin{gathered}
P_{\mathrm{r}}(s)=P_{\mathrm{o}}(s) \frac{1}{f_{\mathrm{r}}(s)} I \xi_{\mathrm{r}}(s) S_{\mathrm{r}}, \\
\Delta P_{\mathrm{ur}}(s)=S_{\mathrm{r}}^{-1} \xi_{\mathrm{r}}^{-1}(s) \Delta P_{\mathrm{u}}(s) \xi_{\mathrm{r}}(s) S_{\mathrm{r}} .
\end{gathered}
$$

Now the nominal plant for our controller design has been reformulated as $P_{\mathrm{r}}(s)$, so that it has a diagonal MRI matrix $f_{\mathrm{r}}(s) I$ and a positive high-frequency gain matrix $\Gamma_{\mathrm{r}}$. The signal $v(t)$ is the new design input for this parameterization, and will be specified later.

Now suppose that $P_{\mathrm{o}}(s)$ is not precisely known but some prior knowledge about its structure may be available. The control objective is to 
design $u_{\mathrm{p}}(t)$ such that the outputs $\hat{y}_{\mathrm{p}}(t)$ of the plant track the outputs $y_{\mathrm{m}}(t)$ of a linear time-invariant reference model described by

$$
y_{\mathrm{m}}(t)=M(s)\left[r_{\mathrm{m}}\right](t),
$$

where $M(s)$ is a stable strictly proper rational matrix and $r_{\mathrm{m}}(t)$ is a uniformly bounded reference input vector. Now, in order to achieve such an objective, we need some assumptions on the modeled part of the plant and the reference model as well as the unmodeled part of the plant. These assumptions heavily depend on the aforcmentioned plant parameterization, and are made in the following.

For the modeled part of the plant and reference model,

(S1) the MRI matrix $\xi_{\mathrm{r}}(s)$ of $P_{\mathrm{o}}(s)$ is known a priori;

(S2) an upper bound $v$ on the observability index of

$$
P_{\mathrm{r}}(s)=P_{\mathrm{o}}(s) \frac{1}{f_{\mathrm{r}}(s)} l \xi_{\mathrm{r}}(s) S_{\mathrm{r}}
$$

is known;

(S3) a matrix $S_{\mathrm{r}}$ such that $K_{\mathrm{r}} S_{\mathrm{r}}=\Gamma_{\mathrm{r}}$ is positivedefinite is known;

(S4) $P_{\mathrm{o}}(s)$ is nonsingular and has stable zeros (minimum phase);

(S5) The degree $\rho$ of $f_{\mathrm{r}}(s)$ is chosen such that $f_{\mathrm{r}}(s) I M(s)$ is proper and stable.

For the unmodeled part of the plant,

(S6) the unmodeled dynamics $\Delta P_{\mathrm{u}}\left(s-k_{1}\right)$ is a strictly proper and stable transfer matrix such that $|D|<a_{1}, \|\left[\Delta P_{\mathrm{u}}\left(s-k_{1}\right) s-D\right](s+$ $\left.a_{2}\right) \|_{\infty}<a_{1}$, for some constants $a_{1}, a_{2}>0$, where $\quad D=\lim _{s \rightarrow \infty} \Delta P_{\mathrm{u}}(s) s \quad$ and $\|X(s)\|_{\infty} \equiv \sup _{w \in \mathbb{R}}|X(\mathrm{j} w)|$ (Tao and Ioannou, 1988);

(S7) the output disturbances are differentiable and the upper bounds on $\left|d_{\mathrm{o}}(t)\right|$ and $\left|(\mathrm{d} / \mathrm{d} t) d_{\mathrm{o}}(\mathrm{t})\right|$ exist.

\section{Remark 2.1.}

- Assumption (S1) is equivalent to the assumption of known relative degree in SISO MRAC systems. It has been shown (Tao and Ioannou, 1988; Singh and Narendra, 1984) that, by designing a precompensator $C(s)$ for $P_{\mathrm{o}}(s)$, the interactor matrix can generically be determined from the relative degree of each entry of $P_{o}(s)$. A systematic approach via network theory has been proposed as an efficient algorithm to determine the optimal precompensator in the sense that the total relative degree is minimum (Asano et al., 1990).

- Assumption (S2) is equivalent to the assumption of a known upper bound on the order of the transfer function in SISO MRAC systems, and is used to parameterize MIMO MRAC systems. It should be noted that this knowledge is necessary to achieve exact model matching in the context of the model reference control when uncertainties are absent.

- Assumption (S3) is equivalent to the assumption of a known sign of the high-frequency gain in SISO MRAC systems, and is used to develop a stable adaptation law.

- The minimum-phase assumption (S4) on the nominal plant $P_{\mathrm{o}}(s)$ is to guarantee internal stability, since the model reference control involves cancellation of the plant zeros. However, as noted by Tao and Ioannou (1988), this assumption does not imply that the overall plant (1) possesses the minimum-phase property.

- The latter part of assumption (S6) is simply to emphasize the fact that $\Delta P_{\mathrm{u}}(s)$ are uncorrelated with $\mu$ in all cases (Ioannou and Tsakalis, 1988). The reasons for assumption (S7) will be clear in the proofs of Theorem 3.1 and 4.1 .

In order to facilitate the presentation of controller design and its subsequent analysis, we now give a definition of 'generalized relative degree' of an MIMO plant based on the discussion of the MRI matrix in Remark 2.1. It is well known that the relative degree of a transfer function $p_{\mathrm{o}}(s)$ can be defined as the degree of the monic polynomial $\xi^{*}(s)$ such that

$$
\lim _{s \rightarrow \infty} p_{o}(s) \xi^{*}(s)=k_{\mathrm{p}} \neq 0 .
$$

A similar idea can now be used to define the generalized relative degree as follows.

Definition 2.1. For a multivariable plant with strictly proper rational transfer matrix $P_{\mathrm{o}}(s)$, the generalized relative degree $(G R D)$ of $P_{\mathrm{o}}(s)$ is defined as the maximum degree of elements of the MRI matrix $\xi_{\mathrm{r}}(s)$ of $P_{\mathrm{o}}(s)$ such that

$$
\lim _{s \rightarrow \infty} P_{\mathrm{o}}(s) \xi_{\mathrm{r}}(s)=K_{\mathrm{r}},
$$

where $K_{\mathrm{r}}$ is nonsingular.

Remark 2.2. According to the parameterization described in Lemma 2.1, the degree of $f_{\mathrm{r}}(s)$ is 
equal to the maximum degree of elements of $\xi_{\mathrm{r}}(s)$. Hence the GRD of the parameterized nominal plant $P_{\mathrm{r}}(s)$ is also equal to that of $P_{\mathrm{o}}(s)$. In other words, the GRD is invariant under the parameterization given by Lemma 2.1 .

\section{3. $M R C$-based error model}

Since the plant parameters are assumed to be unknown, a basic strategy from traditional MRAC (Narendra and Annaswamy, 1988) is now used to construct the error model between $\hat{y}_{\mathrm{p}}$ and $y_{\mathrm{m}}$. Instead of applying the traditional MRAC technique, a new adaptive variable structure control will be given here in order to pursue better robustness and tracking performance. Specifically, when the parameterization (3)-(6) is used, the control inputs are designed in the following form:

$$
\begin{aligned}
v(t)= & \Theta_{0}(t) r(t)+\Theta_{1}^{\mathrm{T}}(t) w_{1}(t) \\
& +\Theta_{2}^{\mathrm{T}}(t) \hat{w}_{2}(t)+\Theta_{3}(t) \hat{y}_{\mathrm{p}}(t), \\
u_{\mathrm{p}}(t)= & \frac{1}{f_{\mathrm{r}}(s)} I \xi_{\mathrm{r}}(s) S_{\mathrm{r}}[v](t),
\end{aligned}
$$

where

$$
\begin{gathered}
\Theta_{1}=\left[\begin{array}{ccc}
\Theta_{11} & \ldots & \Theta_{1, v-1}
\end{array}\right]^{\mathrm{T}}, \\
\Theta_{2}=\left[\begin{array}{lll}
\Theta_{21} & \ldots & \Theta_{2, v-1}
\end{array}\right]^{\mathrm{T}}, \\
\Theta_{0}, \Theta_{3}, \Theta_{i j} \in \mathbb{R}^{n \times n}, \\
w_{1}(t)=\frac{A(s)}{n(s)}[v](t), \\
\hat{w}_{2}(t)=\frac{A(s)}{n(s)}\left[\hat{y}_{\mathrm{p}}\right](t), \\
A(s)=\left[\begin{array}{llll}
I & s I & \ldots & s^{v-2} I
\end{array}\right]^{\mathrm{T}},
\end{gathered}
$$

with $n(s)$ being an arbitrary monic Hurwitz polynomial of degree $v-1$, and $r(t)$ is to be defined shortly. It can be shown (Tao and Ioannou, 1988; Narendra and Annaswamy, 1988) that, when $\mu=0$ and $d_{\mathrm{o}}=0$, constant matrices $\Theta_{i}^{*}, i=0,1,2,3$, exist such that for $\Theta_{i}(t) \equiv \Theta_{i}^{*}$, the closed-loop transfer matrix from $r(t)$ to $y_{\mathrm{p}}(t)$ is equal to $\left[1 / f_{\mathrm{r}}(s)\right] I$. Let the reference model $M(s)$ be chosen such that $f_{\mathrm{r}}(s) I M(s)$ is proper. Then it can be easily verified that $r(t)=$ $f_{\mathrm{r}}(s) I M(s)\left[r_{\mathrm{m}}\right](t)$.

Now, consider the existence of unmodeled dynamics and output disturbances, and decompose the signal $\hat{w}_{2}(t)$ in (8) into two components, $\hat{w}_{2}(t)=w_{2}(t)+w_{2 d_{0}}(t)$, where

$$
\begin{gathered}
w_{2}(t)=\frac{A(s)}{n(s)}\left[y_{\mathrm{p}}\right](t), \\
w_{2 d_{\mathrm{o}}}(t)=\frac{A(s)}{n(s)}\left[d_{\mathrm{o}}\right](t) .
\end{gathered}
$$

Further define the traditional control parameters
$\Theta=\left[\begin{array}{llll}\Theta_{0} & \Theta_{1}^{\mathrm{T}} & \Theta_{2}^{\mathrm{T}} & \Theta_{3}\end{array}\right]^{\mathrm{T}} \in \mathbb{R}^{2 n v \times n}$ and the signal vectors $\hat{w}(t), w(t)$ and $w_{d_{0}}(t)$ as

$$
\hat{w}=\left[\begin{array}{c}
r \\
w_{1} \\
\hat{w}_{2} \\
\hat{y}_{\mathrm{p}}
\end{array}\right]=\left[\begin{array}{c}
r \\
w_{1} \\
w_{2} \\
y_{\mathrm{p}}
\end{array}\right]+\left[\begin{array}{c}
0 \\
0 \\
w_{2 d_{0}} \\
d_{\mathrm{o}}
\end{array}\right]=w+w_{d_{0}} .
$$

One can easily show using the matching condition described above that if unmodeled dynamics and output disturbances exist and $v$ is expressed in an implicit form as

$$
\begin{aligned}
v= & \Theta_{1}^{* \mathrm{~T}} \frac{A(s)}{n(s)}\left\{v+\mu \Delta P_{\mathrm{ur}}(s)[v]\right\} \\
& +\Theta_{2}^{* \mathrm{~T}} \frac{A(s)}{n(s)}\left(\hat{y}_{\mathrm{p}}-d_{\mathrm{o}}\right) \\
& +\Theta_{3}^{*}\left(\hat{y}_{\mathrm{p}}-d_{\mathrm{o}}\right)+\Theta_{\delta}^{*} r-\mu \Delta P_{\mathrm{ur}}(s)[v] \\
= & \Theta^{* \mathrm{~T}} \hat{w}+\Theta_{1}^{* \mathrm{~T}} \frac{A(s)}{n(s)} \mu \Delta P_{\mathrm{ur}}(s)[v] \\
& -\Theta^{* \mathrm{~T}} w_{d_{\mathrm{o}}}-\mu \Delta P_{\mathrm{ur}}(s)[v]
\end{aligned}
$$

then $y_{\mathrm{p}}(t)=M(s)\left[r_{\mathrm{m}}\right](t)$. Hence, from the inputoutput operator point of view, we have

$$
\begin{aligned}
\hat{y}_{\mathrm{p}}(t)= & y_{\mathrm{p}}(t)+d_{\mathrm{o}}(t) \\
= & \frac{1}{f_{\mathrm{r}}(s)} I\left(\Theta _ { 0 } ^ { * - 1 } \left\{v-\Theta^{* \mathrm{~T}} \hat{w}+\Theta^{* \mathrm{~T}} w_{d_{\mathrm{o}}}\right.\right. \\
& +\mu \Delta(s)[v]\}+r)(t)+d_{\mathrm{o}}(t),
\end{aligned}
$$

where

$$
\Delta(s)[v](t)=\left[I-\Theta_{1}^{* \mathrm{~T}} \frac{A(s)}{n(s)}\right] \Delta P_{\mathrm{ur}}(s)[v](t) .
$$

Wc now define the tracking errors $e_{\mathrm{o}}(t)=$ $\hat{y}_{\mathrm{p}}(t)-y_{\mathrm{m}}(t)$, and use (10) with $\Theta_{0}^{*-1}=\Gamma_{\mathrm{r}}$ to obtain the following error model:

$$
\begin{aligned}
e_{\mathrm{o}}(t)= & \frac{1}{f_{\mathrm{r}}(s)} I \Gamma_{\mathrm{s}}\left\{v-\Theta^{* \mathrm{~T}} \hat{w}+\Theta^{* \mathrm{~T}} w_{d_{\mathrm{o}}}\right. \\
& +\mu \Delta(s)[v]\}(t)+d_{\mathrm{o}}(t) .
\end{aligned}
$$

In the following sections, a new adaptive variable structure scheme is proposed for MIMO plants with arbitrary GRD. However, the control structure is much simpler for a GRD-one system, and hence in Section 3 we shall first discuss this class of systems. Based on the analysis for GRD-one systems, the general case can then be presented in a more straightforward manner in Section 4.

\section{THE CASE OF GENERALIZED RELATIVE DEGREE ONE}

When $P_{\mathrm{o}}(s)$ (and hence $P_{\mathrm{r}}(s)$ ) is of GRD-one, the designed Hurwitz polynomial $f_{\mathrm{r}}(s)$ is of first order and the reference model $M(s)$ can be 
chosen to be strictly positive real (SPR) (Narendra and Annaswamy, 1988). The error model (11) can now be rewritten as

$$
\begin{aligned}
e_{\mathrm{o}}(t)= & \frac{1}{f_{\mathrm{r}}(s)} I \Gamma_{\mathrm{r}}\left\{v-\Theta^{* \mathrm{~T}} \hat{w}+\Theta^{* \mathrm{~T}} w_{d_{\mathrm{v}}}\right. \\
& \left.+\mu \Delta(s)[v]+\Theta_{0}^{*} f_{\mathrm{r}}(s)\left[d_{\mathrm{o}}\right]\right\}(\mathrm{t}) .
\end{aligned}
$$

In the error model (12), the terms $\Theta^{* \mathrm{~T}} \hat{w}$, $\Theta^{* \mathrm{~T}} \boldsymbol{w}_{d_{\mathrm{o}}}+\Theta_{0}^{*} f_{\mathrm{r}}(s)\left[d_{\mathrm{o}}\right]$ and $\mu \Delta(s)[v]$ are the uncertainties due to the unknown plant parameters, output disturbances and unmodeled dynamics respectively. Let $\left(A_{\mathrm{m}}, B_{\mathrm{m}}, C_{\mathrm{m}}\right)$ be any minimal realization of $\left[1 / f_{\mathrm{r}}(s)\right] \Pi_{\mathrm{r}}$ that is SPR. Then we can get the following state-space representation of (12):

$$
\begin{aligned}
\dot{e}(t)= & A_{\mathrm{m}} e(t)+B_{\mathrm{m}}\left\{v(t)-\Theta^{* \mathrm{~T}} \hat{w}(t)+\Theta^{* \mathrm{~T}} w_{\mathrm{d}_{\mathrm{o}}}(t)\right. \\
& \left.+\mu \Delta(s)[v](t)+\Theta_{0}^{*} f_{\mathrm{r}}(s)\left[d_{\mathrm{o}}\right](t)\right\}, \\
e_{\mathrm{o}}(t)= & C_{\mathrm{m}} e(t)
\end{aligned}
$$

where the triplet $\left(A_{\mathrm{m}}, B_{\mathrm{m}}, C_{\mathrm{m}}\right)$ satisfies

$$
P_{\mathrm{m}} A_{\mathrm{m}}+A_{\mathrm{m}}^{\mathrm{T}} P_{\mathrm{m}}=-2 Q_{\mathrm{m}}, \quad P_{\mathrm{m}} B_{\mathrm{m}}=C_{\mathrm{m}}^{\mathrm{T}}
$$

for some $P_{\mathrm{m}}=P_{\mathrm{m}}^{\mathrm{T}}>0$ and $Q_{\mathrm{m}}=Q_{\mathrm{m}}^{\mathrm{T}}>0$.

The adaptive variable structure controller for GRD-one plants can now be summarized as follows:

(i) Construct the regressor signal $\hat{w}(t)$ as (9) and the normalization signal $m(t)$ (Ioannou and Tsakalis, 1986) as the state of the system

$$
\begin{gathered}
\dot{m}(t)=-\delta_{0} m(t)+\delta_{1}[|v(t)|+1], \\
m(0)>\frac{\delta_{1}}{\delta_{0}},
\end{gathered}
$$

where $\delta_{0}, \delta_{1}>0$ and $\delta_{0}+\delta_{2}<\min \left(k_{1}, k_{2}\right)$ for some $\delta_{2}>0$. The parameter $k_{2}>0$ is selected such that the poles of $\xi_{\mathrm{r}}^{-1}\left(s-k_{2}\right)$ and the zeros of $n\left(s-k_{2}\right)$ are stable, which is always achievable.

(ii) Design the control signal $v(t)$ as

$$
\begin{aligned}
v(t)= & -\operatorname{sgn}\left[e_{\mathrm{o}}(t)\right]\left[\beta_{1}(t)|\hat{w}(t)|\right. \\
& \left.+\beta_{2}(t)+\beta_{3}(t) m(t)\right]
\end{aligned}
$$

where $\operatorname{sgn}\left(e_{\mathrm{o}}\right)=\left[\operatorname{sgn}\left(e_{\mathrm{o} 1}\right), \quad \operatorname{sgn}\left(e_{\mathrm{o} 2}\right), \ldots\right.$, $\left.\operatorname{sgn}\left(e_{\mathrm{o} n}\right)\right]^{\mathrm{T}}$.

(iii) The adaptation law for the control parameters is given as

$$
\begin{aligned}
& \dot{\beta}_{1}(t)=g_{1}\left|e_{\mathrm{o}}(t)\right||\hat{w}(t)|, \\
& \dot{\beta}_{2}(t)=g_{2}\left|e_{\mathrm{o}}(t)\right|, \\
& \dot{\beta}_{3}(t)=g_{3}\left|e_{\mathrm{o}}(t)\right| m(t),
\end{aligned}
$$

where $g_{j}>0$ is the adaptation gain and $\beta_{f}(0)>0$ (in general, as large as possible) for $j=1,2,3$.

Remark 3.1. The design concept of the adaptive variable structure controller (15), (16) is simply to construct some feedback signals to compensate for the uncertainties for the following reasons:

- with the construction of $m$, it can be shown (Ioannou and Tsakalis, 1986) that $\Delta(s)[v](t) \leq$ $\bar{\gamma} m(t) \forall t \geq 0$ for some constant $\bar{y}>0$;

- using assumption (S7), it can be easily found that $\left|w_{d_{\mathrm{o}}}(t)\right| \leq \bar{\lambda}$ and $\left|f_{\mathrm{r}}(s)\left[d_{\mathrm{o}}\right](t)\right| \leq \bar{d}$ for some positive $\bar{\lambda}, \bar{d}>0$.

Now, we are ready to state our results concerning the properties of global stability, robust property and tracking performance of our new adaptive variable structure scheme with a GRD-one system.

Theorem 3.1. (Global stability, robustness and asymptotic zero tracking performance). Consider the system (3)-(6) satisfying assumptions (S1)(S7), with the degree of $f_{\mathrm{r}}(s)$ being one. If the control input is designed as in (15) and (16) and the adaptation law is chosen as in (17) then there exists $\mu^{*}>0$ such that for $\mu \in\left[0, \mu^{*}\right]$, all signals inside the closed-loop system are bounded and the tracking errors will converge to zero asymptotically.

Proof. Consider the Lyapunov function

$$
V_{\mathrm{a}}=\frac{1}{2} e^{\mathrm{T}} P_{\mathrm{m}} e+\sum_{j=1}^{3} \frac{1}{2 g_{j}}\left(\beta_{j}-\beta_{j}^{*}\right)^{2},
$$

where $P_{\mathrm{m}}$ satisfies $(14)$, and $\beta_{1}^{*}=\left|\Theta^{*}\right|, \quad \beta_{2}^{*}=$ $\left|\Theta^{*}\right| \bar{\lambda}+\left|\Theta_{0}^{*}\right| \bar{d}$ and $\beta_{3}^{*}=\mu^{*} \bar{\gamma}$, with $\mu^{*}$ defined in the Appendix. Then the time derivative of $V_{\mathrm{a}}$ along the trajectory (13), (17) will be

$$
\begin{aligned}
\dot{V}_{\mathrm{a}}= & -e^{\mathrm{T}} Q_{\mathrm{m}} e+e_{\mathrm{o}}^{\mathrm{T}}\left\{v-\Theta^{* \mathrm{~T}} \hat{w}+\Theta^{* \mathrm{~T}} w_{d_{o}}\right. \\
& \left.+\mu \Delta(s)[v]+\Theta_{0}^{*} f_{\mathrm{r}}(s)\left[d_{\mathrm{o}}\right]\right\}+\sum_{j=1}^{3} \frac{1}{g_{j}}\left(\beta_{j}-\beta_{j}^{*}\right) \dot{\beta}_{j} \\
\leq & -e^{\mathrm{T}} Q_{\mathrm{m}} e-\left|e_{\mathrm{o}}\right|\left(\beta_{1}-\beta_{i}^{*}\right)|\hat{w}| \\
& -\left|e_{\mathrm{o}}\right|\left(\beta_{2}-\beta_{2}^{*}\right)-\left|e_{\mathrm{o}}\right|\left(\beta_{3}-\beta_{3}^{*}\right) m \\
& +\sum_{j=1}^{3} \frac{1}{g_{j}}\left(\beta_{j}-\beta_{j}^{*}\right) \dot{\beta}_{j} \\
\leq & -q_{\mathrm{m}}|e|^{2}
\end{aligned}
$$

for some constant $q_{\mathrm{m}}>0$. This implies that $e \in L_{2} \cap L_{\infty}$ and $\beta_{1}, \beta_{2}, \beta_{3}, e_{0} \in L_{\infty}$, and hence all signals inside the closed-loop system are bounded owing to Lemma A.1 in the Appendix. On the other hand, it can be concluded that $\dot{e} \in L_{\infty}$ by (13). Hence $e \in L_{2} \cap L_{\infty}$ and $\dot{e} \in L_{\infty}$ 
readily imply that $e$ and $e_{\mathrm{o}}$ will at least converge to zero asymptotically by Barbalat's lemma (Narendra and Annaswamy, 1988).

In Theorem 3.1, a suitable integral adaptation law is given to compensate for the unavailable knowledge of the bounds on $\beta_{j}^{*}, j=1,2,3$. Theoretically, the adaptive variable structure controller will stabilize the closed-loop system with guaranteed robustness and asymptotic zero tracking performance no matter what the $\beta_{j}(0)$ are. However, according to the following theorem, we expect that positive and large values of $\boldsymbol{\beta}_{j}(0)$ should result in better transient response and tracking performance, especially when $\beta_{j}(0)>\beta_{j}^{*}$.

Theorem 3.2. (Finite-time zero tracking performance with high-gain design). Consider the system setup in Theorem 3.1. If $\beta_{j}(0) \geq \beta_{j}^{*}$, $j=1,2,3$, then the output tracking errors will converge to zero in finite time, with all signals inside the closed-loop system remaining bounded.

Proof. Consider the Lyapunov function $V_{\mathrm{b}}=$ $\frac{1}{2} e^{\mathrm{T}} P_{\mathrm{m}} e$, where $P_{\mathrm{m}}$ satisfies (14). The time derivative of $V_{\mathrm{b}}$ along the trajectory (13) becomes

$$
\begin{aligned}
\dot{V}_{\mathrm{b}}= & -e^{\mathrm{T}} Q_{\mathrm{m}} e-\left|e_{\mathrm{o}}\right|\left(\beta_{1}-\beta_{1}^{*}\right)|\hat{w}| \\
& -\left|e_{\mathrm{o}}\right|\left(\beta_{2}-\beta_{2}^{*}\right)-\left|e_{\mathrm{o}}\right|\left(\beta_{3}-\beta_{3}^{*}\right) m \\
\leq & -e^{\mathrm{T}} Q_{\mathrm{m}} e \\
\leq & -k_{3} V_{\mathrm{b}}
\end{aligned}
$$

for some $k_{3}>0$, since $\beta_{j}(t) \geq \beta_{j}^{*} \forall t \geq 0$. This implies that $e$ approaches zero at least exponentially fast. Furthermore, from the fact that

$$
\begin{aligned}
e_{\mathrm{o}}^{\mathrm{T}} \Gamma_{\mathrm{r}}^{-1} \dot{e}_{\mathrm{o}}= & e_{\mathrm{o}}^{\mathrm{T}} \Gamma_{\mathrm{r}}^{-1}\left(C_{\mathrm{m}} A_{\mathrm{m}} e+C_{\mathrm{m}} B_{\mathrm{m}}\left\{v-\Theta^{* \mathrm{~T}} \hat{w}\right.\right. \\
& \left.\left.+\Theta^{* \mathrm{~T}} w_{d_{\mathrm{o}}}+\mu \Delta(s)[v]+\Theta_{0}^{*} f_{\mathrm{r}}(s)\left[d_{\mathrm{o}}\right]\right\}\right) \\
\leq & \left|e_{\mathrm{o}}\right|\left[k_{4}|e|-\left(\beta_{1}|\hat{w}|+\beta_{2}\right.\right. \\
& \left.\left.+\beta_{3} m-\beta_{1}^{*}|\hat{w}|-\beta_{2}^{*}-\beta_{3}^{*} m\right)\right],
\end{aligned}
$$

where $k_{4}=\left|\Gamma_{\mathrm{r}}^{-1} C_{\mathrm{m}} A_{\mathrm{m}}\right|$, and the fact that $|e|$ approaches zero at least exponentially fast, there exists a finite time $T_{1}>0$ such that $e_{\mathrm{o}}^{\mathrm{T}} \Gamma_{\mathrm{r}}^{-1} \dot{e}_{\mathrm{o}} \leq$ $-k_{5}\left|e_{\mathrm{o}}\right|$ for all $t>T_{1}$ and for some $k_{5}>0$. Note that

$$
e_{\mathrm{o}}^{\mathrm{T}} \Gamma_{\mathrm{r}}^{-1} \dot{e}_{\mathrm{o}}=\frac{1}{2 \mathrm{~d}} \frac{1}{\mathrm{~d} t}\left(e_{\mathrm{o}}^{\mathrm{T}} \Gamma_{\mathrm{r}}^{-1} e_{\mathrm{o}}\right)=\frac{1}{2} \frac{\mathrm{d}}{\mathrm{d} t}\left(e_{\mathrm{o}}^{\mathrm{T}} U^{\mathrm{T}} e_{\mathrm{o}}\right),
$$

where $U$, satisfying $U^{\mathrm{T}} U=\frac{1}{2}\left(\Gamma_{\mathrm{r}}^{-1}+\Gamma_{\mathrm{r}}^{-\mathrm{T}}\right)$, is symmetric positive-definite, so that

$$
\frac{1}{2} \frac{\mathrm{d}}{\mathrm{d} t}\left|U e_{\mathrm{o}}\right|^{2} \leq-k_{5}\left|e_{\mathrm{o}}\right| \leq-\frac{k_{5}}{|U|}\left|U e_{\mathrm{o}}\right|<0
$$

for all $t \geq T_{1}$ when $\left|U e_{\mathrm{o}}\right| \neq 0$. This implies that the sliding surfaces $U e_{\mathrm{o}}=0$ are guaranteed to be reached in some finite time $T_{2}>T_{1}>0$. Then the nonsingularity of the matrix $U$ readily implies the finite-time convergence of the output tracking errors $e_{\mathrm{o}}$.

Remark 3.2. Although theoretically only asymptotic zero tracking performance is achieved when the initial control parameters are chosen arbitrarily one is encouraged to set the adaptation gain $g_{j}$ in (17) as large as possible. This is because large adaptation gains will provide high adaptation speed, and hence increase the control parameters to a suitable level of magnitude so as to achieve a satisfactory performance as quickly as possible. These expected results can be observed in the simulation examples, and some comments will also be addressed in that section.

\section{THE CASE OF ARBITRARY GENERALIZED RELATIVE DEGREE}

When the GRD of $P_{\mathrm{o}}(s)$ is greater than one, the reference model $M(s)$ is no longer SPR, so that the controller design becomes more complicated. The adaptive variable structure control scheme for a multivariable system (1) (or equivalently (3)) with $G R D \geq 2$ that satisfies assumptions (S1)-(S7) is now designed as follows.

\section{Systematic design procedures}

(i) Choose an operator $L_{1}(s)=l_{1}(s) \cdots$ $l_{\rho-1}(s)=\left(s+\alpha_{1}\right) \cdots\left(s+\alpha_{\rho-1}\right)$ such that $\left[1 / f_{\mathrm{r}}(s)\right] L_{1}(s)$ is SPR.

(ii) Define the augmented signal

$$
y_{\mathrm{a}}(t)=\frac{1}{f_{\mathrm{r}}(s)} L_{1}(s) I\left[-v_{1}+\frac{1}{L_{1}(s)} I[v]\right](t)
$$

and auxiliary errors

$$
\begin{aligned}
e_{\mathrm{a} 1}(t) & =e_{\mathrm{o}}(t)-y_{\mathrm{a}}(t) \\
e_{\mathrm{a} 2}(t) & =-\left[v_{1}\right]_{\mathrm{av}}+\frac{1}{l_{1}(s)} I\left[v_{2}\right](t), \\
& \vdots \\
e_{\mathrm{a} \rho}(t) & =-\left[v_{\rho-1}\right]_{\mathrm{av}}+\frac{1}{l_{\rho-1}(s)} I\left[v_{\rho}\right](t),
\end{aligned}
$$

where $\left[v_{i}\right]_{\mathrm{av}}$ is the average control of $v_{i}$, given by

$$
\left[v_{i}\right]_{\mathrm{av}}=\frac{1}{F(\tau s)}\left[v_{i}\right]=\frac{1}{(\tau s+1)^{2}}\left[v_{i}\right]
$$

with $\tau>0$ being small enough. The reason 
why $\tau$ has to be chosen sufficiently small will be clear in Theorem 4.1 and its proof.

(iii) Design the control signals $v, v_{i}, i=$ $1, \ldots, \rho$, as follows:

$$
\begin{aligned}
v_{i}(t)= & -\operatorname{sgn}\left[e_{\mathrm{a} i}(t)\right]\left[\beta_{i 1}(t)\left|\hat{\xi}_{i}(t)\right|\right. \\
& \left.+\beta_{i 2}(t)+\beta_{i 3}(t) m(t)\right], \\
v(t)= & v_{\rho}(t),
\end{aligned}
$$

where

$$
\begin{aligned}
\hat{\xi}_{1}(t)= & \frac{1}{l_{1}(s)} \cdots \frac{1}{l_{n-1}(s)} I[\hat{w}](t) \\
= & \frac{1}{L_{1}(s)} I[\hat{w}](t), \\
\hat{\xi}_{i}(t)= & \frac{1}{F(\tau s)} l_{i-1}(s) I\left[\hat{\xi}_{i-1}\right](t), \\
& i=2, \ldots, \rho, \\
\dot{m}(t)= & -\delta_{3} m(t)+\delta_{4}(|v(t)|+1), \\
& m(0)>\frac{\delta_{4}}{\delta_{3}},
\end{aligned}
$$

with $\quad \delta_{3}, \delta_{4}>0 \quad$ and $\quad \delta_{3}+\delta_{5}<$ $\min \left(k_{1}, k_{2}, \alpha_{1}, \ldots, \alpha_{\rho-1}, 1 / \tau\right)$ for some $\delta_{5}>0$.

(iv) Finally, the adaptation laws for the control parameters $\beta_{i 1}, \beta_{i 2}, \beta_{i 3}, i=1, \ldots, \rho, \quad$ are given by

$$
\begin{aligned}
& \dot{\beta}_{i 1}(t)=g_{i 1}\left|e_{\mathrm{a} i}(t)\right|\left|\hat{\xi}_{i}(t)\right|, \\
& \dot{\beta}_{i 2}(t)=g_{i 2}\left|e_{\mathrm{a} i}(t)\right|, \\
& \dot{\beta}_{i 3}(t)=g_{i 3}\left|e_{\mathrm{a} i}(t)\right| m(t),
\end{aligned}
$$

where $g_{i j}>0$ and $\beta_{i j}(0)>0, i=1, \ldots, \rho$, $j=1,2,3$.

In the following discussions, the construction of the feedback signals $\hat{\xi}_{1}, \ldots, \hat{\xi}_{\rho}, m$ and the controller (20) will be clear.

In order to analyze the tracking performance and the closed-loop stability of the proposed adaptive variable structure controller, we first rewrite the error equation (11) as

$$
\begin{aligned}
e_{\mathrm{o}}(t)= & \frac{1}{f_{\mathrm{r}}(s)} I\left(v+\Gamma_{\mathrm{r}}\left\{-\Theta^{* \mathrm{~T}} \hat{w}+\Theta^{* \mathrm{~T}} w_{d_{\mathrm{o}}}\right.\right. \\
& \left.+\mu \Delta(s)[v]\}+\Gamma_{\Delta} v\right)(t)+d_{\mathrm{o}}(t)
\end{aligned}
$$

where $\Gamma_{\mathrm{r}} \triangleq I+\Gamma_{\Delta}$. Note that there is one more uncertain term $\Gamma_{\Delta} v$ appearing in the error model (22), which represents the unavailable knowledge of the high-frequency gain matrix $K_{\mathrm{r}}$ (and hence $\left.\Gamma_{\mathrm{r}}\right)$. Second, denote $L_{i}(s)=$ $l_{i}(s) \cdots l_{\rho-1}(s)=\left(s+\alpha_{i}\right) \cdots\left(s+\alpha_{\rho-1}\right)$ for $i=$ $1, \ldots, \rho$ and $L_{\rho}(s)=1$. According to the design of the above auxiliary errors (19) and error model (22), we readily find that $e_{\mathrm{a} 1}$ always satisfies

$$
\begin{aligned}
e_{\mathrm{a} 1}(t)= & \frac{1}{f_{\mathrm{r}}(s)} L_{1}(s) I\left(v_{1}-\Gamma_{\mathrm{r}} \Theta^{* \mathrm{~T}} \hat{\xi}_{1}\right. \\
& +\frac{1}{L_{1}(s)}\left\{\Gamma_{\mathrm{r}} \Theta^{* \mathrm{~T}} w_{d_{\mathrm{o}}}+f_{\mathrm{r}}(s) I\left[d_{\mathrm{o}}\right]\right\} \\
& \left.+\frac{1}{L_{1}(s)}\left\{\mu \Gamma_{\mathrm{r}} \Delta(s)[v]+\Gamma_{\Delta} v\right)\right)(t) .
\end{aligned}
$$

The average control $\left[v_{1}\right]_{\mathrm{av}}$ in (15) can now be obtained by operating on (23) with $1 / F(\tau s)$, so that $e_{\mathrm{a} 2}$ satisfies

$$
\begin{aligned}
e_{\mathrm{a} 2}(t)= & \frac{1}{l_{1}(s)} I\left(v_{2}-\Gamma_{\mathrm{r}} \Theta^{* \mathrm{~T}} \hat{\xi}_{2}+\frac{1}{F(\tau s) L_{2}(s)}\right. \\
& \times\left\{\Gamma_{\mathrm{r}} \Theta^{* \mathrm{~T}} w_{d_{\mathrm{o}}}+f_{\mathrm{r}}(s) I\left[d_{\mathrm{o}}\right]\right\}+\frac{1}{F(\tau s) L_{2}(s)} \\
& \left.\times\left\{\mu \Gamma_{\mathrm{r}} \Delta(s)[v]+\Gamma_{\Delta} v\right\}-\varepsilon_{1}\right)(t)
\end{aligned}
$$

where

$$
\varepsilon_{1}=\frac{1}{F(\tau s) L_{2}(s)} f_{\mathrm{r}}(s)\left[e_{a 1}\right] .
$$

By the same token, we can deduce that

$$
\begin{aligned}
e_{\mathrm{a} i}(t)= & \frac{1}{l_{i-1}(s)} I\left(v_{i}-\Gamma_{\mathrm{r}} \Theta^{*} \mathrm{~T} \hat{\xi}_{i}+\frac{1}{F^{i-1}(\tau s) L_{i}(s)}\right. \\
& \times\left\{\Gamma_{\mathrm{r}} \Theta^{* \mathrm{~T}} w_{d_{\mathrm{o}}}+f_{\mathrm{r}}(s) I\left[d_{\mathrm{o}}\right]\right\}+\frac{1}{F^{i-1}(\tau s) L_{i}(s)} \\
& \left.\times\left\{\mu \Gamma_{\mathrm{r}} \Delta(s)[v]+\Gamma_{\Delta} v\right\}-\varepsilon_{i-1}\right)(t)
\end{aligned}
$$

for $i=3, \ldots, \rho$, where

$$
\begin{aligned}
\varepsilon_{i-1}= & \frac{1}{F(\tau s)} l_{i-1}(s)\left[\varepsilon_{i-2}\right] \\
& +\frac{1}{F(\tau s)} l_{i-1}(s) l_{i-2}(s)\left[e_{a i-1}\right] .
\end{aligned}
$$

It should be noted that all the auxiliary errors are now explicitly expressed as the output terms of some linear systems with SPR transfer matrix $\left(\left[1 / f_{\mathrm{r}}(s)\right] L_{1}(s) I\right.$ or $\left.\left[1 / l_{i-1}(s)\right] I\right)$ deiven by some uncertain signals due to the unavailable knowledge of plant parameters, high-frequency gain matrix, unmodeled dynamics and output disturbances.

Remark 4.1. The construction of the variable 
structure controller $(20)$ is now clear, since the following hold:

- $\frac{1}{L_{1}(s)}\left(\mu \Gamma_{\mathrm{r}} \Delta(s)+\Gamma_{\Delta}\right)$ and

$$
\frac{1}{F^{i-1}(\tau s) L_{i}(s)}\left(\mu \Gamma_{\mathrm{r}} \Delta(s)+\Gamma_{\Delta}\right)
$$

$i=2, \ldots, \rho$,

are strictly proper stable with known stability margins,

- $\frac{1}{L_{1}(s)}\left(\Gamma_{\mathrm{r}} \Theta^{* \mathrm{~T}} w_{d_{\mathrm{o}}}+f_{\mathrm{r}}(s) I\left[d_{\mathrm{o}}\right]\right)$ and

$$
\frac{1}{F^{i-1}(\tau s) L_{i}(s)}\left(\Gamma_{\mathrm{r}} \Theta^{* \mathrm{~T}} w_{d_{\mathrm{o}}}+f_{\mathrm{r}}(s) I\left[d_{\mathrm{o}}\right]\right),
$$

$i=2, \ldots, \rho$,

are bounded owing to assumption (S7).

Therefore the terms in $e_{a i}, i=1, \ldots, \rho$, will satisfy

$$
\begin{aligned}
\left|\Gamma_{\mathrm{r}} \Theta^{* \mathrm{~T}} \hat{\xi}_{i}\right| & \leq \beta_{i 1}^{*}\left|\hat{\xi}_{i}\right|, \\
\left|\frac{1}{F^{i-1}(\tau s) L_{i}(s)}\left(\Gamma_{\mathrm{r}} \Theta^{* \mathrm{~T}} w_{d_{\mathrm{o}}}+f_{\mathrm{r}}(s) I\left[d_{\mathrm{o}}\right]\right)\right| & \leq \beta_{i 2}^{*}, \\
\left|\frac{1}{F^{i-1}(\tau s) L_{i}(s)}\left(\mu \Gamma_{\mathrm{r}} \Delta(s)[v]+\Delta \Gamma_{\mathrm{r}} v\right)\right| & \leq \beta_{i 3}^{*} m
\end{aligned}
$$

for some suitably defined constants $\beta_{i 1}^{*}>0$, $\beta_{i 2}^{*}>0$ and $\beta_{i 3}^{*}>0, i=1, \ldots, \rho$.

The results described in Remark 4.1 show that the same techniques for the controller design of a GRD-one system can now be used for auxiliary errors $e_{\mathrm{a} 1}, \ldots, e_{\mathrm{a} \rho}$. But what happens to the real output errors? In Theorem 4.1, we summarize the main results of the systematically designed adaptive variable structure controller for unknown multivariable plants with $G R D \geq 2$.

Theorem 4.1. (Global stability, robustness and asymptotic tracking performance.) Consider the system set-up in Theorem 3.1 but with $P_{\mathrm{o}}(s)$ being GRD $\rho \geq 2$. If the adaptive variable structure controller is designed as in (18)-(21) then there exist $\tau^{*}$ and $\mu^{*}$ such that for all $\tau \in\left(0, \tau^{*}\right]$ and $\mu \in\left[0, \mu^{*}\right]$, the following hold:

(i) all signals inside the closed-loop system remain bounded;

(ii) the auxiliary errors $e_{\mathrm{a} i}, i=1, \ldots, \rho$, converge asymptotically to zero;

(iii) the output tracking errors $e_{\mathrm{o}}$ converge asymptotically to a residual set whose size depends on the design parameter $\tau$.

Proof. The proof consists of three parts.

Part I. We prove the boundedness of $e_{a i}$ and $\beta_{i j}, i=1, \ldots, \rho, j=1,2,3$.

Step 1. First, consider the auxiliary error $e_{\mathrm{a} 1}$, which satisfies $(23)$. Since $\left[1 / f_{\mathrm{r}}(s)\right] L_{1}(s) I$ is SPR, we have the following realization of (23):

$$
\begin{aligned}
\dot{e}_{1}= & A_{1} e_{1}+B_{1}\left(v_{1}-\Gamma_{\mathrm{r}} \Theta^{* \mathrm{~T}} \hat{\xi}_{1}\right. \\
& +\frac{1}{L_{1}(s)} I\left\{\Gamma_{\mathrm{r}} \Theta^{* \mathrm{~T}} w_{d_{\mathrm{o}}}+f_{\mathrm{r}}(s) I\left[d_{\mathrm{o}}\right]\right\} \\
& \left.+\frac{1}{L_{1}(s)} I\left\{\mu \Gamma_{\mathrm{r}} \Delta(s)[v]+\Gamma_{\Delta} v\right\}\right), \\
e_{\mathrm{a} 1}= & C_{1} e_{1},
\end{aligned}
$$

where $A_{1}^{\mathrm{T}} P_{1}+P_{1} A_{1}=-Q_{1}$ and $P_{1} B_{1}=C_{1}^{\mathrm{T}}$ for some $P_{1}=P_{1}^{\mathrm{T}}>0$ and $Q_{1}>0$. If we choose a Lyapunov function similar to $V_{\mathrm{a}}$ in Theorem 3.1, i.e.

$$
V_{1}=\frac{1}{2} e_{1}^{\mathrm{T}} P_{1} e_{1}+\sum_{j=1}^{3} \frac{1}{2 g_{1 j}}\left(\beta_{1 j}-\beta_{1 j}^{*}\right)^{2},
$$

and apply the control law (20) and the adaptation law (21), then for some $q_{1}>0$,

$$
\begin{aligned}
\dot{V}_{1}= & -e_{1}^{\mathrm{T}} Q_{1} e_{1}+e_{\mathrm{a} 1}^{\mathrm{T}}\left(v_{1}-\Gamma_{\mathrm{r}} \Theta^{* \mathrm{~T}} \hat{\xi}_{1}\right. \\
& +\frac{1}{L_{1}(s)} I\left\{\Gamma_{\mathrm{r}} \Theta^{* \mathrm{~T}} w_{d_{\mathrm{o}}}+f_{\mathrm{r}}(s) I\left[d_{\mathrm{o}}\right]\right\} \\
& \left.+\frac{1}{L_{1}(s)} I\left\{\mu \Gamma_{\mathrm{r}} \Delta(s)[v]+\Gamma_{\Delta} v\right\}\right) \\
& +\sum_{j=1}^{3} \frac{1}{g_{1 j}}\left(\beta_{1 j}-\beta_{1 j}^{*}\right) \dot{\beta}_{1 j} \\
\leq & -q_{1}\left|e_{1}\right|^{2}-\left|e_{\mathrm{a} 1}\right|\left[\left(\beta_{11}-\beta_{11}^{*}\right)\left|\hat{\xi}_{1}\right|\right. \\
& \left.+\left(\beta_{12}-\beta_{12}^{*}\right)+\left(\beta_{13}-\beta_{13}^{*}\right) m\right] \\
& +\sum_{j=1}^{3} \frac{1}{g_{1 j}}\left(\beta_{1 j}-\beta_{1 j}^{*}\right) \dot{\beta}_{1 j} \\
\leq & -q_{1}\left|e_{1}\right|^{2} .
\end{aligned}
$$

This implies that $e_{\mathrm{a} 1} \in L_{2} \cap L_{\infty}$ and $\beta_{11}, \beta_{12}$, $\beta_{13} \in L_{\infty}$.

Step 2. From (24), $e_{\mathrm{a} 2}$ satisfies

$$
\begin{aligned}
\dot{e}_{\mathrm{a} 2}= & -\alpha_{1} e_{\mathrm{a} 2}+\left(v_{2}-\Gamma_{\mathrm{r}} \Theta^{* \mathrm{~T}} \hat{\xi}_{2}\right. \\
& +\frac{1}{F(\tau s) L_{2}(s)}\left\{\Gamma_{\mathrm{r}} \Theta^{* \mathrm{~T}} w_{d_{\mathrm{o}}}+f_{\mathrm{r}}(s) I\left[d_{\mathrm{o}}\right]\right\} \\
& \left.+\frac{1}{F(\tau s) L_{2}(s)}\left\{\mu \Gamma_{\mathrm{r}} \Delta(s)[v]+\Gamma_{\Delta} v\right\}-\varepsilon_{1}\right)
\end{aligned}
$$


Note that $\left|\varepsilon_{1}\right| \leq \bar{\varepsilon}^{*}$ for some $\bar{\varepsilon}^{*}>0$, since $e_{\mathrm{al}}$ is bounded by the result in Step 1 . Now choose the Lyapunov function

$$
\begin{aligned}
V_{2}= & \frac{1}{2} e_{\mathrm{a} 2}^{\mathrm{T}} e_{\mathrm{a} 2}+\frac{1}{2 g_{21}}\left(\beta_{21}-\beta_{21}^{*}\right)^{2}+\frac{1}{2 g_{22}}\left(\beta_{22}-\beta_{22}^{*}\right)^{2} \\
& +\frac{1}{2 g_{23}}\left(\beta_{23}-\beta_{23}^{*}-\bar{\varepsilon}^{*}\right)^{2},
\end{aligned}
$$

so that we can again show that $\dot{V}_{2} \leq-e_{a 2}^{\mathrm{T}} e_{\mathrm{a} 2} \leq 0$ if the control law in (20) and the update law in (21) are applied. This leads to the conclusion that $e_{\mathrm{a} 2} \in L_{2} \cap L_{\infty}$ and $\beta_{21}, \beta_{22}, \beta_{23} \in L_{\propto}$.

Step $i$. Since for $3 \leq i \leq \rho$,

$$
\begin{aligned}
\dot{e}_{\mathrm{a} i}= & -\alpha_{i, 1} e_{\mathrm{a} i}+\left(v_{i}-\Gamma_{\mathrm{r}} \Theta^{* \mathrm{~T}} \hat{\xi}_{i}+\frac{1}{F^{i-1}(\tau s) L_{i}(s)}\right. \\
& \times\left\{\Gamma_{\mathrm{r}} \Theta^{* \mathrm{~T}} w_{d_{\mathrm{o}}}+f_{\mathrm{r}}(s) I\left[d_{\mathrm{o}}\right]\right\}+\frac{1}{F^{i-1}(\tau s) L_{i}(s)} \\
& \left.\times\left\{\mu \Gamma_{\mathrm{r}} \Delta(s)[v]+\Gamma_{\Delta} v\right\}-\varepsilon_{i-1}\right)
\end{aligned}
$$

and $\left|\varepsilon_{i-1}\right| \leq \bar{\varepsilon}^{*}$ (with some abuse of notation), the Lyapunov function

$$
\begin{aligned}
V_{i}= & \frac{1}{2} e_{\mathrm{a} i}^{\mathbf{T}} e_{\mathrm{a} i}+\frac{1}{2 g_{i 1}}\left(\beta_{i 1}-\beta_{i 1}^{*}\right)^{2}+\frac{1}{2 g_{i 2}}\left(\beta_{i 2}-\beta_{i 2}^{*}\right)^{2} \\
& +\frac{1}{2 g_{i 3}}\left(\beta_{i 3}-\beta_{i 3}^{*}-\bar{\varepsilon}^{*}\right)^{2}
\end{aligned}
$$

can be used to guarantee $\dot{V}_{i} \leq-e_{\mathrm{a} a}^{\mathrm{T}} e_{\mathrm{a} i} \leq 0$ if the control law in (20) and the update law in (21) are applied. Consequently, $e_{\mathrm{a} i} \in L_{2} \cap L_{\infty}$ and $\boldsymbol{\beta}_{i 1}, \beta_{i 2}, \beta_{i 3} \in L_{\infty}$.

Part II. We prove the boundedness of all signals inside the closed-loop. Define $\bar{e}_{\mathrm{a} i}=$ $\left[1 / f_{\mathrm{r}}(s)\right] L_{i-1}(s) I\left[e_{\mathrm{a} i}\right], i=2, \ldots, \rho$ and $E_{\mathrm{a}}=e_{\mathrm{a} 1}+$ $\bar{e}_{\mathrm{a} 2}+\cdots+\bar{e}_{\mathrm{a} \rho}$, which is bounded owing to the boundedness of $e_{\mathrm{a} i}$ guaranteed in Part I. Then it can be derived from (19) that

$$
\begin{aligned}
E_{\mathrm{a}}= & e_{\mathrm{o}} \\
+ & \frac{1}{f_{\mathrm{r}}(s)} L_{1}(s) I\left(v_{1}-\frac{1}{L_{1}(s)} I[v]\right) \\
& +\frac{1}{f_{\mathrm{r}}(s)} L_{1}(s) I\left(-\left[v_{1}\right]_{\mathrm{av}}+\frac{1}{l_{1}(s)} I\left[v_{2}\right]\right) \\
& \vdots \\
& +\frac{1}{f_{\mathrm{r}}(s)} L_{\rho-1}(s) I\left(-\left[v_{\rho-1}\right]_{\mathrm{av}}+\frac{1}{l_{\rho-1}(s)} I[v]\right) \\
= & e_{\mathrm{o}}+\left(1-\frac{1}{F(\tau s)}\right) \frac{L_{1}(s)}{f_{\mathrm{r}}(s)} I\left(v_{1}+\frac{1}{l_{1}(s)} I\left[v_{2}\right]\right. \\
& \left.+\cdots+\frac{1}{l_{1}(s) \cdots l_{\rho-2}(s)} I\left[v_{\rho-1}\right]\right) \\
\triangleq & e_{\mathrm{o}}+R
\end{aligned}
$$

Now, since $\left\|(v)_{t}\right\|_{\infty} \leq \kappa\left\|\left(e_{0}\right)_{t}\right\|_{\infty}+\kappa$ by Lemma
A.1 in the Appendix, it can be easily found from the definition of $v_{i}$ that

$$
\begin{aligned}
& \|\left(v_{1}+\frac{1}{l_{1}(s)} I\left[v_{2}\right]+\cdots\right. \\
& \left.\quad+\frac{1}{l_{1}(s) \cdots l_{\rho-2}(s)} I\left[v_{\rho-2}\right]\right)\left\|_{\imath}\right\|_{\infty} \leq \kappa\left\|\left(e_{0}\right)_{\imath}\right\|_{\infty}+\kappa .
\end{aligned}
$$

Furthermore, since

$$
\begin{gathered}
\left\|\frac{1}{s}\left(1-\frac{1}{F(\tau s)}\right)\right\|_{\infty}=2 \tau, \\
\left\|\frac{s L_{1}(s)}{f_{\mathbf{r}}(s)}\right\|_{\infty}=\kappa \text { for some } \kappa>0,
\end{gathered}
$$

we can conclude that

$$
\begin{aligned}
\left\|(R)_{t}\right\|_{\infty} \leq & \left\|\frac{1}{S}\left(1-\frac{1}{F(\tau s)}\right)\right\|_{\infty}\left\|\frac{s L_{1}(s)}{f_{\mathrm{r}}(s)}\right\|_{\infty} \\
& \times\left[\kappa\left\|\left(e_{\mathrm{o}}\right)_{t}\right\|_{\infty}+\kappa\right] \leq \tau\left(\kappa\left\|\left(e_{\mathrm{o}}\right)_{t}\right\|_{\infty}+\kappa\right) .
\end{aligned}
$$

Now, from (26), we have

$$
\begin{aligned}
\left\|\left(e_{\mathrm{o}}\right)_{t}\right\|_{\infty} & \leq\left\|\left(E_{\mathrm{a}}\right)_{t}\right\|_{\infty}+\left\|(R)_{t}\right\|_{\infty} \\
& \leq\left\|\left(E_{\mathrm{a}}\right)_{t}\right\|_{\infty}+\tau\left[\kappa\left\|\left(e_{\mathrm{o}}\right)_{t}\right\|_{\infty}+\kappa\right],
\end{aligned}
$$

which implies that there exists a $\tau^{*}>0$ such that $1-\tau^{*} \kappa>0$ and for all $\tau \in\left(0, \tau^{*}\right)$,

$$
\left\|\left(e_{\mathrm{o}}\right)_{t}\right\|_{\infty} \leq \frac{\left\|\left(E_{\mathrm{a}}\right)_{t}\right\|_{\infty}+\tau \kappa}{1-\tau \kappa} .
$$

This implies that the output errors are bounded. Finally, combining Lemma A.1 and (27), we readily conclude that all signals inside the closed-loop system remain bounded.

Part III. We investigate the tracking performance of $e_{\mathrm{a} i}$ and $e_{\mathrm{o}}$. Since all signals inside the closed-loop system are bounded, we have

$$
e_{\mathrm{a} i} \in L_{2} \cap L_{\infty}, \quad \dot{e}_{\mathrm{a} i} \in L_{\infty}, \quad i=1, \ldots, \rho .
$$

Hence, by Barbalat's lemma, $e_{\mathrm{a} i}$ approaches zero asymptotically. Since $e_{a i}$ reaches zero asymptotically and $E_{\mathrm{a}}=e_{\mathrm{a} 1}+\bar{e}_{\mathrm{a} 2}+\cdots+\bar{e}_{\mathrm{a} \rho}$, it can be concluded that $E_{\text {a }}$ will also converge asymptotically to zero. It is now clear from (27) that $e_{\mathrm{o}}$ converges asymptotically to a small residual set whose size depends on the design parameter $\tau$.

As discussed in Theorem 3.2, if the initial choices of control parameters $\beta_{i j}(0)$ satisfy the high-gain conditions $\beta_{i j}(0) \geq \beta_{i j}^{*}$ then, by using the same argument as given in the proof of Theorem 3.2, we can guarantee the exponential convergent behavior and finite-time tracking performance of all the auxiliary errors $e_{\mathrm{a} i}$. Since $e_{a i}$ reaches zero in some finite time and $E_{\mathrm{a}}=e_{\mathrm{a} 1}+\bar{e}_{\mathrm{a} 2}+\cdots+\bar{e}_{\mathrm{a} \rho}$, it can be concluded 
that $E_{\mathrm{a}}$ converges exponentially to zero and $e_{\mathrm{o}}$ converges to a small residual set whose size depends on the design parameter $\tau$. We now summarize the results in the following theorem.

Theorem 4.2. (Exponential tracking performance with high-gain design.) Consider the system setup in Theorem 4.1. If $\beta_{i j}(0) \geq \beta_{i i}^{*}$, $i=1,2, \ldots, \rho, j=1,2,3$, then there exist $\tau^{*}$ and $\mu^{*}$ such that for all $\tau \in\left(0, \tau^{*}\right]$ and $\mu \in\left[0, \mu^{*}\right]$, the following hold:

(i) all signals inside the closed-loop system remain bounded;

(ii) the auxiliary errors $e_{\mathrm{a} i}, i=1, \ldots, \rho$, converge to zero in finite time;

(iii) the output tracking errors $e_{\mathrm{o}}$ converge exponentially to a residual set whose size depends on the design parameter $\tau$.

Remark 4.2. As to the upper bound on the feasible value of $\tau$, it can actually be obtained through a laborious derivation following the steps in the proof of Theorem 4.1. However, in that case the bound will be over-conservative. Therefore it may be better to apply some intelligent prior trial-and-error procedure to obtain a suitable $\tau$.

\section{COMPUTER SIMULATIONS}

\subsection{Application to an aircraft model (AIRC)}

The adaptive variable structure scheme is now applied to a realistic model taken from the Appendix in Maciejowski (1989). It represents a linearized model of the vertical-plane dynamics of an aircraft, which is minimum-phase with three inputs, three outputs and five states. The state-space representation of this AIRC model is

$$
A=\left[\begin{array}{rrrrr}
0 & 0 & 1.1320 & 0 & -1.0000 \\
0 & -0.0538 & -0.1712 & 0 & 0.0705 \\
0 & 0 & 0 & 1.0000 & 0 \\
0 & 0.0485 & 0 & -0.8556 & -1.0130 \\
0 & -0.2909 & 0 & 1.0532 & -0.6859
\end{array}\right] \text {, }
$$

$$
B=\left[\begin{array}{rrr}
0 & 0 & 0 \\
-0.1200 & 1.0000 & 0 \\
0 & 0 & 0 \\
4.4190 & 0 & -1.665 \\
1.5750 & 0 & -0.0732
\end{array}\right] \text {, }
$$

$$
C=\left[\begin{array}{lllll}
1 & 0 & 0 & 0 & 0 \\
0 & 1 & 0 & 0 & 0 \\
0 & 0 & 1 & 0 & 0
\end{array}\right] \text {. }
$$

It can be found, using a Matlab software package, that the MRI matrix and high- frequency matrix are

$$
\begin{aligned}
\xi_{\mathrm{r}}(s) & =\left[\begin{array}{ccc}
(s+2) & \frac{25}{3}(s+2)^{2} & 0 \\
0 & (s+2)^{2} & 0 \\
0 & 0 & (s+2)^{2}
\end{array}\right], \\
K_{\mathrm{r}} & =\left[\begin{array}{ccc}
0 & -13.1250 & 0.0732 \\
-0.12 & 0.9249 & -0.0051 \\
0 & 4.4190 & -1.6650
\end{array}\right] .
\end{aligned}
$$

Hence a controller design for a GRD-two system is used in this example. Now suppose that there are uncertainties

$$
\begin{aligned}
& \Delta \mathrm{P}_{\mathrm{u}}(s)= \\
& {\left[\begin{array}{ccc}
\frac{4}{s+8} & \frac{s+3}{s^{2}+11 s+30} & \frac{6}{s+7} \\
\frac{1}{s+9} & \frac{3}{s+12} & \frac{2 s+9}{s^{2}+17 s+70} \\
\frac{s+6}{s^{2}+13 s+40} & \frac{4}{s+7} & \frac{5}{s+12}
\end{array}\right],}
\end{aligned}
$$

present in this system. The parameterization of (3) is now used, with the following design:

- design parameters

$$
\begin{aligned}
S_{\mathrm{r}} & =K_{\mathrm{r}}^{-1}, \\
n(s) & =s+10 \\
f_{\mathrm{r}}(s) & =(s+7)(s+9), \\
L_{1}(s) & =l_{1}(s)=s+8, \\
F(\tau s) & =\left(\frac{1}{20 \pi} s+1\right)^{2}
\end{aligned}
$$

- augmented signal and auxiliary errors

$$
\begin{aligned}
y_{\mathrm{a}}(t) & =\frac{1}{f_{\mathrm{r}}(s)} L_{1}(s) I\left(-v_{1}+\frac{1}{L_{1}(s)} I[v]\right)(t), \\
e_{\mathrm{a} 1}(t) & =e_{\mathrm{o}}(t)-y_{\mathrm{a}}(t), \\
e_{\mathrm{a} 2}(t) & =-\left[v_{1}\right]_{\mathrm{av}}+\frac{1}{l_{1}(s)} I[v](t) ;
\end{aligned}
$$

- controller

$$
\begin{aligned}
v_{i}(t)= & -\operatorname{sgn}\left[e_{a i}(t)\right]\left[\beta_{i 1}(t)\left|\hat{\xi}_{i}(t)\right|\right. \\
& \left.+\beta_{i 2}(t)+\beta_{i 3}(t) m(t)\right], \quad i=1,2, \\
v(t)= & v_{2}(t), \\
\dot{m}(t)= & -0.1 m(t)+0.01[|v(t)|+1], \quad m(0)=0.2 ;
\end{aligned}
$$

- adaptation law

$$
\begin{aligned}
& \dot{\beta}_{i 1}(t)=g_{i 1}\left|e_{\mathrm{ai}}(t)\right|\left|\hat{\xi}_{i}(t)\right|, \\
& \dot{\beta}_{i 2}(t)=g_{i 2}\left|e_{\mathrm{ai}}(t)\right|, \\
& \dot{\beta}_{i 3}(t)=g_{i 3}\left|e_{\mathrm{ai}}(t)\right| m(t) ;
\end{aligned}
$$

- reference model and reference input

$$
\begin{aligned}
M(s) & =\frac{63}{f_{\mathrm{r}}(s)} I=\frac{63}{(s+7)(s+9)} I \\
r_{\mathrm{m}}(t) & =\left[\begin{array}{lll}
\sin t & 2-3 \cos t
\end{array}\right]^{\mathrm{T}}, \quad r(t)=63 r_{\mathrm{m}}(t) .
\end{aligned}
$$

Three simulation cases are studied extensively in this example in order to verify all the theoretical results and corresponding comments. All the 
cases will assume that there are initial output perturbations $\hat{y}_{\mathrm{p}}(0)-y_{\mathrm{m}}(0)=\left[\begin{array}{llll}-1.6 & 1.1 & 4.5\end{array}\right]^{\mathrm{T}}$.

(i) In the first case, we arbitrarily choose the initial control parameters $\beta_{i j}(0)$ as

$$
\begin{array}{lll}
\beta_{11}(0)=3, & \beta_{12}(0)=3, & \beta_{13}(0)=0.5, \\
\beta_{21}(0)=1, & \beta_{22}(0)=1, & \beta_{23}(0)=0.5,
\end{array}
$$

and set all the adaptation gains $g_{i j}=1$. As shown in Figs 1(a-c) (the time trajectorics of $\hat{y}_{\mathrm{p}}$ and $y_{\mathrm{m}}$ ), the global stability, robustness and asymptotic tracking performance are achieved, but some undesirable transient behaviors between $\hat{y}_{\mathrm{p}}$ and $y_{\mathrm{m}}$ are also observed. However, as shown in Figs 1(d, e) (the time trajectories of the control parameters), the large tracking errors in the transient period result in rapid increases in the magnitudes of the control parameters $\beta_{i j}$, and after the transient period the tracking performance is then satisfied.

(ii) In the second case, we want to demonstrate the effectiveness of a proper choice of $\beta_{i j}(0)$, and we repeat the previous simulation case by increasing the values of $\beta_{i j}(0)$ to

$$
\begin{aligned}
& \beta_{11}(0)=10, \quad \beta_{12}(0)=10, \quad \beta_{13}(0)=1, \\
& \beta_{21}(0)=5, \quad \beta_{22}(0)=5, \quad \beta_{23}(0)=1 \text {. }
\end{aligned}
$$

The nice transient and tracking performance between $\hat{y}_{\mathrm{p}}$ and $y_{\mathrm{m}}$ can be observed in Figs 2(a-c).

(iii) As noted in Remark 3.2, if there is no easy way to estimate suitable initial control parameters $\beta_{i j}(0)$ like those in the second simulation case, it is suggested that one use large adaptation gains in order to increase the adaptation rate of the control parameters $\beta_{i j}$ such that the nice transient and tracking performance as described in case (ii) can be retained to some extent. Hence, in this case, we use the initial control parameters as in case (i) but set all the adaptation gains to $g_{i j}=5$. The expected results are shown in Fig. 3, where rapid increases in the control parameters do lead to satisfactory transient and tracking performance.

\subsection{Simulations of AIRC model using MRAC} with switching $\sigma$ modification

In order to make a comparison with the traditional MRAC scheme, we repeat the simulations by using the MRAC scheme with switching $\sigma$ modification (Tao and Ioannou, 1988). This control scheme is now briefly described as follows:

- controller

$$
\begin{aligned}
v(t)= & \Theta_{0}(t) r(t)+\Theta_{1}^{\mathrm{T}}(t) w_{1}(t) \\
& +\Theta_{2}^{\mathrm{T}}(t) \hat{w}_{2}(t)+\Theta_{3}(t) \hat{y}_{p}(t) \\
= & \Theta^{\mathrm{T}}(t) \hat{w}(t), \\
u_{\mathrm{p}}(t)= & \frac{1}{f_{\mathrm{r}}(s)} I \xi_{\mathrm{r}}(s) S_{\mathrm{r}}[v](t),
\end{aligned}
$$

where $\Theta(t)$ and $\hat{w}(t)$ are defined as in (7) and (8);

- adaptation law

$$
\begin{gathered}
\dot{\Theta}^{\mathrm{T}}(t)=-K_{1}^{-\mathrm{T}} \frac{\epsilon_{1}(t) \zeta^{\mathrm{T}}(t)}{m^{2}(t)}-\sigma_{1} \Theta^{\mathrm{T}}(t), \\
\dot{\psi}_{\mathrm{o}}(t)=-K_{2} \frac{\epsilon_{1}(t) \xi^{\mathrm{T}}(t)}{m^{2}(t)}-\sigma_{2} \psi_{\mathrm{o}}(t),
\end{gathered}
$$

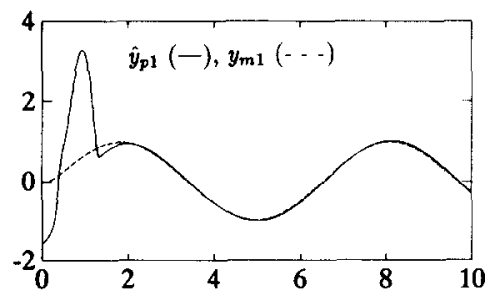

(a) time (sec)

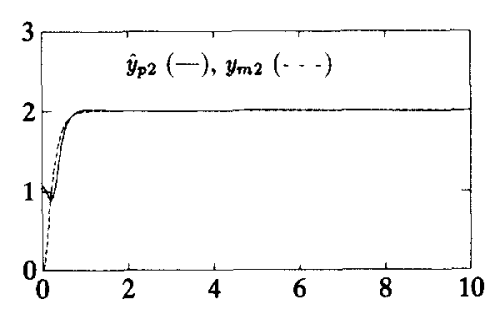

(b) time (sec)

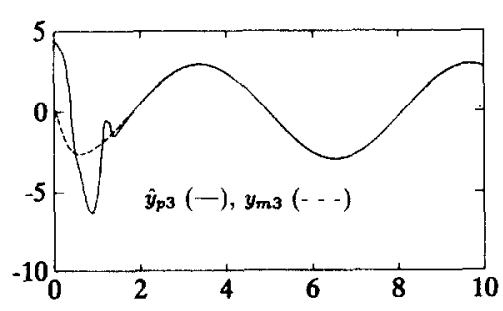

(c) time (sec)

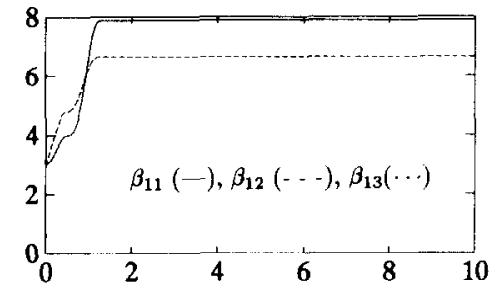

(d) time (sec)

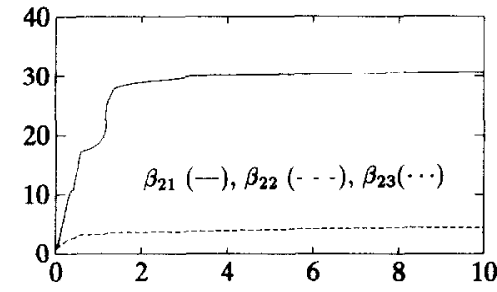

(e) time (sec)

Fig. 1. Tracking performances with small initial control parameters and small adaptation gains. 


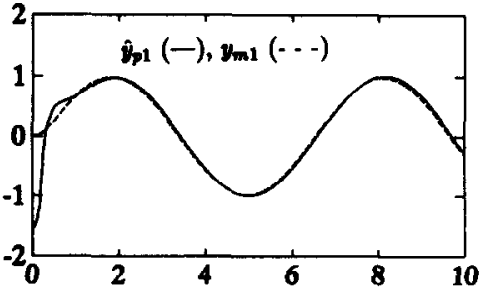

(a) time (sec)

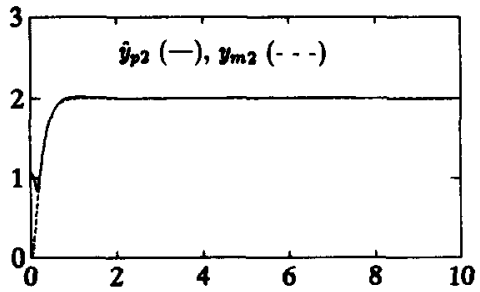

(b) time (sec)

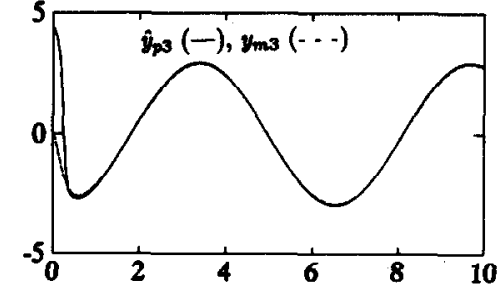

(c) time (sec)

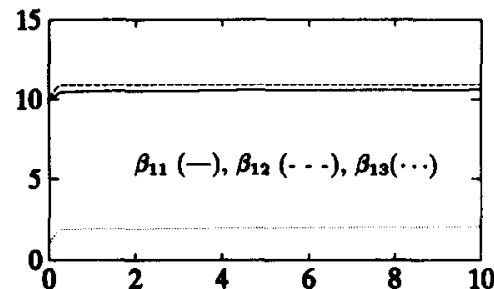

(d) time (sec)

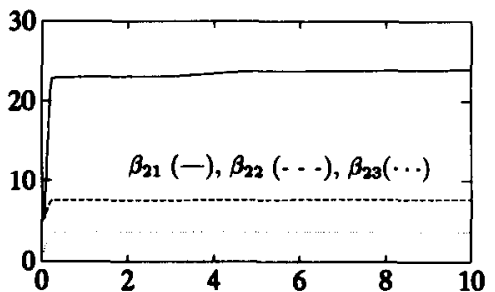

(e) time (sec)

Fig. 2. Tracking performances with large initial control parameters and small adaptation gains.

where $K_{1}$ and $K_{2}$ are positive-definite matrices, $m(t)$ is the normalization signal (15), $\sigma_{1}$ and $\sigma_{2}$ are the switching $\sigma$ - modification parameters, $\epsilon_{1}(t)$ is the measured estimation error, and $\zeta(t)$ and $\xi(t)$ are some regressor signals (for details, see Tao and Ioannou, 1988).

All the necessary design parameters are the same as those in Section 5.1, except for the initial control parameters $\Theta(0)=0$ and $\psi_{0}(0)=0$. The output tracking performances are shown in Fig. 4. Note that in order to achieve an acceptable tracking accuracy, the time interval shown in Fig. 4 is $[0,100]$.

\section{Discussion.}

(i) In Section 5.1, we have studied the effects of the proper choice of the initial control parameters and the adaptation gains on the tracking performances. Theoretically, the larger the $\beta_{i j}(0)$ (especially $\left.\beta_{i j}(0) \geq \beta_{i j}^{*}\right)$, the better are the tracking performances. But large $\beta_{i j}(0)$ (i.e. high gain) would likely cause input saturation, which is a common problem in MRAC systems, especially using variable structure design. However, in our simulations, we have found that there often exist some levels of magnitude of the control parameters that yield satisfactory performances, and these levels are often less than the theoretically required bounds $\beta_{i j}^{*}$ in most cases. In other words, the upper bounds on the control parameters $\beta_{i j}^{*}$ derived in this paper are in general too conservative. Hence an intelligent approach

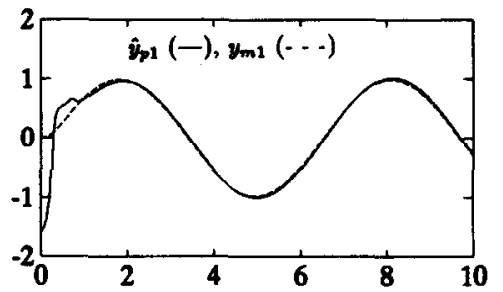

(a) time (sec)

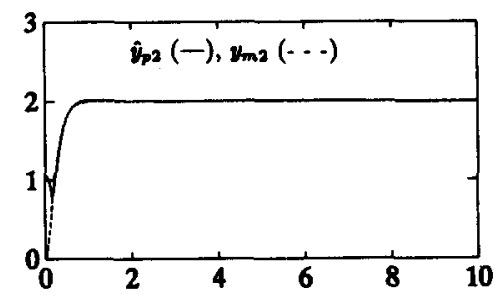

(b) time (sec)

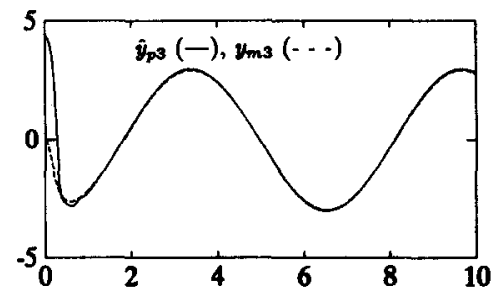

(c) time (sec)

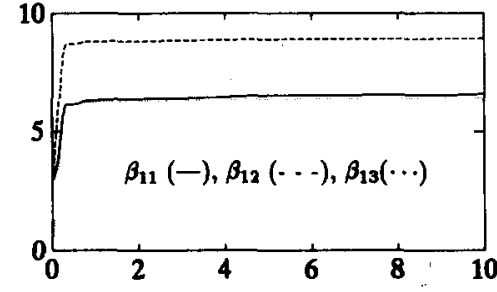

(d) time (sec)

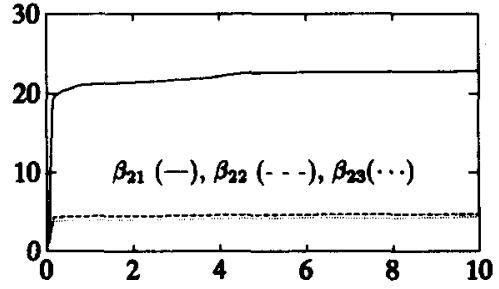

(e) time (sec)

Fig. 3. Tracking performances with small initial control parameters and large adaptation gains. 


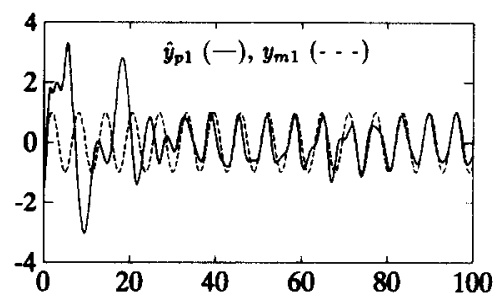

(a) time (sec)

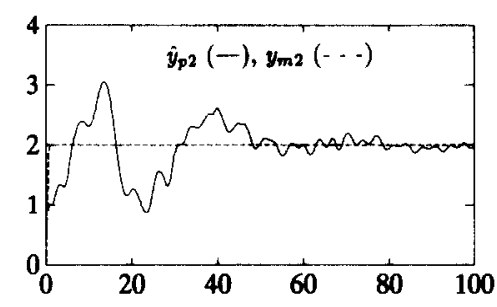

(b) time (sec)

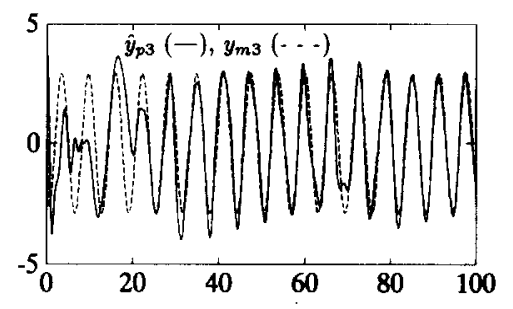

(c) time (sec)

Fig. 4. Tracking performances of traditional MRAC.

to get both satisfactory performances and reasonable values of control parameters is the idea presented in the third simulation case, where a combination of small initial control parameters and high adaptation gains is used. The reasonable values of the control parameters will now be determined by the adaptation process. In the second and third simulation cases, we find that the tracking performances are almost the same, although the initial control parameters $\beta_{i j}(0)$ in case (ii) are much larger than those in case (iii). It is interesting to note that with the aid of high adaptation gains, the control parameters in case (iii) reach some levels of magnitude quickly in order to guarantee the desired tracking performance. Despite this, from Figs 2(d,e) and 3(d,e), we have found that the levels of magnitude reached by the adaptation process in case (iii) are smaller than those in case (ii).

(ii) In the proposed adaptive variable structure controller, the initial choice of control parameters $\beta_{i j}(0)$ and the design of adaptation gains $g_{i j}$ really reflect the improvement in tracking performances. In this scheme, in spite of the systematic design procedure, an easy rule of thumb for a guaranteed performance is to set the $\beta_{i j}(0)$ or adaptation gains $g_{i j}$ as large as possible (especially $\beta_{i j}(0) \geq \beta_{i j}^{*}$ ). In traditional MRAC schemes, it is expected that the tracking perforances might be improved when the initial choices of control parameters $\Theta(0)$ and $\psi_{0}(0)$ are closer to $\Theta^{*}$ and $\psi_{\mathrm{o}}^{*}$. However, it is hard to accurately estimate these true control parameters $\Theta^{*}$ and $\psi_{\mathrm{o}}^{*}$, especially in the MIMO case. So there is no easy way to choose $\Theta(0)$ and $\psi_{0}(0)$ before controller design, and this is why we have simply set $\Theta(0)=0$ and $\psi_{\mathrm{o}}(0)=0$ in the above simulation. Another approach to improve the tracking perfor- mances in traditional MRAC schemes is persistent excitation of the input signal, which is not practical in most control cases and is not necessary in our proposed scheme.

(iii) It is interesting to note that the total number of control parameters updated in the proposed adaptive variable structure scheme is $3 \rho$ for a GRD- $\rho$ plant, which is far less than in traditional MRAC scheme. Taking the simulation case in this section for example, we have $n$ (the input/output numbers) $=3, \quad v$ (the upper bound on observability index) $=2$ and $\rho$ (the generalized relative degree) $=2$, so that the total numbers of control parameters used are $3 \rho=6$ in our scheme and $(2 \times n \times v) \times$ $(n)+n \times n=45$ in the traditional scheme.

(iv) For this example, we have used other possible type of unmodeled dynamics $\Delta P_{\mathrm{u}}(s)$ different from (28) for simulation, and have found that the tracking performances remained unchanged so long as $\Delta P_{\mathrm{u}}(s)$ satisfies assumption (S6). But, as indicated in the proof in the Appendix and Part II of Theorem 4.1, closed-loop stability will depend on the strength of the unmodeled dynamics, i.e. the value of $\mu$. Taking this application for example, we have found that the tracking performances can still be retained when $\mu<0.1$, but closed-loop stability is gradually lost as $\mu$ increases, and the system even becomes unstable when $\mu>1.4$. A possible reason is that the plant, including modeled and unmodeled dynamics, becomes nonminimum-phase when $\mu$ is large, so that the required growth rate condition (A.1) cannot be achieved. However, it is hard to estimate the allowed bound $\mu^{*}$ given in the Appendix In this paper, we only guarantee that the proposed adaptive variable 
structure controller is robust to a class of unmodeled dynamics up to some strength.

\section{CONCLUSIONS}

A new adaptive variable structure scheme has been proposed for MRAC problems for uncertain multivariable plants. The main contribution of this paper is the complete version of adaptive variable structure design for solving the robustness and performance of multivariable MRAC problems. A detailed analysis of the closed-loop stability and tracking performance has been given. It has been shown that without any persistent excitation the output tracking errors can be driven to zero for GRD-one systems and driven asymptotically to a small residual set for systems with any higher GRD. Furthermore, under a suitable choice of initial conditions on the control parameters, the tracking performances can be improved, which is hardly achievable with traditional MRAC schemes, especially for multivariable plants with uncertainties.

Acknowledgement-This work has been supported by the National Science Council, R.O.C. under Grant NSC 82-0422E-002-045.

\section{REFERENCES}

Asano, T., K. Yoshikawa and S. Suzuki (1990). A network-theoretic approach to the design of a precompensator for multivariable adaptive control. IEEE Trans. Autom. Control, AC-35, 706-710.

Chien, C. J. and L. C. Fu (1992). A new robust model reference control with improved performance for a class of multivariable unknown plants. Int. J. Adaptive Control and Sig. Process., 6, 69-93.

Costa, R. R. and L. Hsu (1992). Robustness of VS-MRAC with respect to unmodeled dynamics and external disturbances. Int. J. Adaptive Control and Sig. Process., 6, 19-33.

Das, M. (1986). Multivariable adaptive model matching using less a priori information. J. Dyn. Syst., Meas. and Control, 108, 151-153.

Desoer, C. A. and M. Vidyasagar (1975). Feedback Systems: Input-Output Properties. Academic Press, New York.

Dion, J. M., L. Dugard and J. Carrillo (1988). Interactor and multivariable adaptive model matching. IEEE Trans. Autom. Coritrol, AC-33, 399-401.

Elliott, H. and W. A. Wolovich (1982). A parameter adaptive control structure for linear multivariable systems. IEEE Trans. Autom. Control, AC-27, 340-351.

Elliott, H. and W. A. Wolovich (1984). Parameterization issues in multivariable adaptive control. Automatica, 20, 533-545.

Filippov, A. F. (1964). Differential equations with discontinuous right-hand side. Am. Math. Soc. Transl., 42, 199-231.

Fu, L. C. (1991). A robust model reference adaptive control using variable structure adaptation for a class of plants. Int. J. Control, 35, 1359-1375.

Fu, L. C. (1992). A new robust MRAC using variable structure design for relative degree two plants. Automatica, 28, 911-925.

Hsu, L. (1990). Variable structure model-reference adaptive control (VS-MRAC) using only input output measure ments: the general case, IEEE Trans. Autom. Control, AC-35, 1238-1243.
Hsu, L. and R. R. Costa (1989). Variable structure model reference adaptive control using only input and output measurement: Part 1. Int. J. Control, 49, 339-416.

Ioannou, P. A. and K. S. Tsakalis (1986). A robust direct adaptive control. IEEE Trans. Autom. Control, AC-31, 1033-1043.

Ioannou, P. A. and K. S. Tsakalis (1988). The class of unmodeled dynamics in robust adaptive control. In Proc. American Control Conf., Atlanta, GA, pp. 337-342.

Maciejowski, J. M. (1989). Multivariable Feedback Design. Addison-Wesley, Reading, MA.

Miller, D. E. and E. J. Davison (1991). An adaptive controller that provides an arbitrarily good transient and steady-state response. IEEE Trans. Autom. Control, AC-36, 68-81.

Narendra, K. S. and A. M. Annaswamy (1988). Stable Adaptive Systems. Prentice-Hall, Englewood Cliffs, NJ.

Narendra, K. S. and J. D. Bðsković (1992). A combined direct, indirect and variable structure method for robust adaptive control. IEEE Trans. Autom. Control, AC-37, 262-268.

Sastry, S. S. and M. Bodson (1989). Adaptive Control: Stability, Convergence, and Robustness, Prentice-Hall, Englewood Cliffs, NJ.

Singh, R. P. and K. S. Narendra (1984). Prior information in the design of multivariable adaptive controllers. IEEE Trans. Aut. Control, AC-28, 1108-1111.

Tao, G. and P. A. Ioannou (1988). Robust model reference adaptive control for multivariable plants. Int. J. Adaptive Control and Sig. Process., 2, 217-248.

Tao, G. and P. A. loannou (1989). An MRAC for multivariable plants with zero residual tracking error. In 28th IEEE Proc. Conf. on Decision and Control, Tampa, FL, pp. 1597-1600.

Wolovich, W. A. and P. F. Falb (1976). Invariants and canonical forms under dynamic compensation. SIAM $J$. Control Optim., 14, 996-1008.

\section{APPENDIX}

Lemma A.1. Consider the controller design in Theorem 3.1 or 4.1. If the control parameters $\beta_{j}$ or $\beta_{i j}, i=1, \ldots, \rho, j=$ $1,2,3$, are uniformly bounded then there exists $\mu^{*}>0$ such that $v(t)$ satisfies

$$
\left\|(v)_{t}\right\|_{\infty} \leq \kappa\left\|\left(e_{\infty}\right)_{t}\right\|_{\infty}+\kappa
$$

for all $\mu \in\left[0, \mu^{*}\right], t \geq 0$, where $\kappa$ is used here and below to denote any suitably defined positive constant.

Proof. Since $y_{\mathrm{p}}(t)=P_{\mathrm{r}}(s)\left[I+\mu \Delta P_{\mathrm{ur}}(s)\right][v](t)$, the following equation will hold without considering the exponentially decaying signals from initial conditions:

$$
y_{\mathrm{P}}(t)=P_{\mathrm{r}}(s) f_{\mathrm{r}}(s) I\left[I+\mu \Delta P_{\mathrm{ur}}(s)\right] \frac{1}{f_{\mathrm{r}}(s)} I[v](t) .
$$

Note that $f_{\mathrm{r}}(s) I$ is the MRI matrix of $P_{\mathrm{r}}(s)$, so that $P_{\mathrm{r}}(s) f_{\mathrm{r}}(s) I$ is a proper rational transfer matrix and has stable zeros. Hence, let $v_{\mathrm{f}}(t)=\left[1 / f_{\mathrm{r}}(s)\right] I[v](t)$. Then (A.2) is in fact equivalent to

$$
v_{\mathrm{f}}(t)=\frac{1}{f_{\mathrm{r}}(s)} I P_{\mathrm{r}}^{-1}(s)\left[y_{\mathrm{p}}\right](t)-\mu \Delta P_{\mathrm{ur}}(s)\left[v_{\mathrm{f}}\right](t),
$$

which, by the small-gain theorem (Desoer and Vidyasagar, 1975), implies that there exists $\mu^{*}>0$ such that

$$
\begin{aligned}
\left\|\left(v_{\mathrm{f}}\right)_{t}\right\|_{\infty} & \leq \kappa\left\|\left(y_{\mathrm{p}}\right)_{t}\right\|_{\infty}+\kappa \\
& \leq \kappa\left\|\left(e_{\mathrm{o}}\right)_{t}\right\|_{\infty}+\kappa
\end{aligned}
$$

for all $\mu \in\left[0, \mu^{*}\right]$. However, one can easily observe that

$$
v_{i j}=\frac{1}{f_{r}(s)}\left[v_{i}\right], \quad j=1,2, \ldots, n,
$$


where $v_{i j}$ and $v_{j}$ denote the $j$ th elements of $v_{f}$ and $v$ respectively. It is clear that if we can show that $v_{j}$ is bounded by $v_{i j}$ then (A.1) is achieved.

By using Lemma 2.8 of Narendra and Annaswamy (1988), the key point to show the boundedness between $v_{f j}$ and $v_{j}$ from (A.5) is the growing behavior of the signal $v_{j}$. The above statement can be expressed more precisely as follows: if $v_{j}$ satisfies

$$
\left|v_{i}\left(t_{1}\right)\right| \geq c_{2}\left|v_{i}\left(t_{1}+T\right)\right|,
$$

where $t_{1}$ and $t_{1}+T$ are the time instants defined as

$$
\left[t_{1}, t_{1}+T\right] \subset \Omega=\left\{t:\left|v_{j}(t)\right|=\left\|\left(v_{j}\right)_{t}\right\|_{\infty}\right\},
$$

and $c_{2}$ is a constant $\in(0,1)$, then $v_{j}$ will be bounded by $v_{\mathrm{f}}$ Now, in order to establish (A.6) and (A.7), let $\left(A_{\mathrm{p}}, B_{\mathrm{p}}, C_{\mathrm{p}}\right)$ and $(\Lambda, B)$ be the state-space realizations of $P_{\mathrm{r}}(s)[I+$ $\left.\mu \Delta P_{\mathrm{ur}}(s)\right]$ and $A(s) / n(s)$ respectively. Also define $Z=$
$\left[\begin{array}{llll}x_{\mathrm{p}}^{\mathrm{T}} & \hat{w}_{1}^{\mathrm{T}} & \boldsymbol{w}_{2}^{\mathrm{T}} & m\end{array}\right]^{\mathrm{T}}$. Then, using the augmented system

$$
\begin{aligned}
\frac{\mathrm{d}}{\mathrm{d} t}\left[\begin{array}{c}
x_{\mathrm{p}} \\
\hat{w}_{1} \\
w_{2} \\
m
\end{array}\right]= & {\left[\begin{array}{cccc}
A_{\mathrm{p}} & 0 & 0 & 0 \\
0 & \Lambda & 0 & 0 \\
B C_{\mathrm{p}} & 0 & \Lambda & 0 \\
0 & 0 & 0 & -\delta_{0}
\end{array}\right]\left[\begin{array}{l}
x_{\mathrm{p}} \\
\hat{w}_{1} \\
w_{2} \\
m
\end{array}\right] } \\
& +\left[\begin{array}{c}
B_{\mathrm{p}} \\
B \\
0 \\
0
\end{array}\right] v+\left[\begin{array}{c}
0 \\
0 \\
0 \\
\delta_{1}
\end{array}\right](|v|+1),
\end{aligned}
$$

we can show from the definition of $v$ in (16) or (20) that

$$
|\dot{Z}| \leq \kappa\left\|(Z)_{t}\right\|_{\infty}+\kappa .
$$

This means that $Z$ is regular (Sastry and Bodson, 1989), so that $\hat{w}, m$ and $y_{p}$ will grow at most exponentially fast (if unbounded), which in turn guarantees (A.6) and (A.7). This completes our proof. 